\title{
Evaluación de la recarga en los acuíferos de las formaciones Salto Chico, Hernandarias y Ubajay. Arroyo Palmar, Entre Ríos, Argentina
}

\author{
Adrián Silva Busso ${ }^{(1,2)}$, Luis Moreno Merino ${ }^{(3)}$ y Mario Chury ${ }^{(2)}$ \\ (1)Dpto, de Cs, Geológicas, FCEN, UBA Pabellón 2, Ciudad Universitaria, Buenos Aires (1407), Argentina. silvabusso@ \\ yahoo.com.ar \\ (2)GIGAM, Universidad Tecnológica Nacional - Regional Concordia (UTN-FRCON), Salta 277, Concordia, Entre Ríos \\ Argentina.mchury@gmail.com \\ ${ }^{(3)}$ Instituto Geológico Minero de España, Ríos Rosas 23, 28003 Madrid, España. I.moreno @igme.es
}

\begin{abstract}
RESUMEN
El estudio de los mecanismos y volúmenes de recarga de los acuíferos es de vital importancia para una explotación sostenible del recurso subterráneo, tiene además una gran incidencia en la productividad agrícola, principal actividad económica en Argentina. Sin embargo, los estudios de recarga son tan escasos como necesarios en este país. Se ha realizado un detallado estudio edáfico e hidrogeológico en una cuenca hidrológica tipo en una zona agrícola característica de la región pampeana. Se ha tomado un intenso registro climático, de humedad de suelo y cargas hidráulicas a partir de estaciones diseñadas para este fin. Se ha calculado la magnitud de la recarga vertical a partir de un balance de agua en la zona no saturada, demostrando que las condiciones geológicas de la cuenca son el principal factor que determina su valor, así, donde aflora la $\mathrm{F}$. Ubajay la recarga neta calculada es del $1,41 \%$ de las precipitaciones totales, mientras que donde aflora la $\mathrm{F}$. Hernandarias no llega al $0,04 \%$, valores atípicos en la región pampeana argentina. Este escenario hace pensar que la recarga vertical no es un proceso dominante ni cuantitativamente importante, y se aleja mucho de los valores anteriormente propuestos que estimaban que la recarga en la región oscilaba entre un $7 \%$ y un $15 \%$ de las precipitaciones medias anuales. Las implicaciones desde el punto de vista de la gestión del agua de estos nuevos datos pueden ser muy notables y obligan a replantearse el modelo conceptual de funcionamiento de los acuíferos en esta región.
\end{abstract}

Palabras Clave: Acuíferos, Entre Ríos, Infiltración, Recarga

\section{Evaluation of aquifer recharge into Salto Chico, Hernandarias and Ubajay formations. Palmar Creek, Entre Ríos, Argentina}

\begin{abstract}
The study of recharge mechanisms and volumes in aquifers is one of the most important issues for achieving sustainable exploitation of the groundwater resource. This factor has an impact on agricultural productivity as irrigation increases the yields of many crops and agriculture is the main economic activity in Argentina. However, recharge studies are not common in the country and this is why this paper is a new contribution. We have carried out a detailed study of the soil and hydrogeology in a hydrological basin. This basin is in an agricultural area typical of the Pampean region. Detailed records of precipitation, soil moisture and hydraulic head have been collected from stations designed for this purpose. We have also analyzed the influence and magnitude of vertical recharge from a water balance in the unsaturated zone. The calculation of the net recharge has also been included in the saturated zone. We have shown that the geological conditions of the basin influence the aquifer recharge. In outcrop zone of $F$. Ubajay the infiltration of surpluses allows a vertical transit equivalent to $6.61 \%$ with net recharge values of $1.41 \%$ of precipitation. In the outcrop zone of $F$. Hernandarias the conditions do not favour the infiltration of water surpluses and only allow a transit equivalent to $0.89 \%$ with a net recharge of $0.04 \%$ of precipitation. This scenario suggests that vertical recharge is not a dominant or quantitatively important process, which is atypical in Argentina's Pampean region. These values are far from those previously established which varied between $7 \%$ and $15 \%$ of the average annual rainfall. The im-
\end{abstract}


plications for water management in the region are significant and make it necessary to rethink the conceptual model of the aquifers in the area

Key words: recharge; aquifers; Entre Ríos; infiltration

\section{Introduction}

The study of recharge mechanisms and volumes in aquifers is one of the most important issues for achieving sustainable exploitation of the groundwater resource. This factor has an impact on agricultural productivity as irrigation increases the yields of many crops. In Argentina agriculture is the main economic activity. However, the recharge studies are not common in the country and this is why this paper is a new contribution. We have carried out a detailed study of the soils and hydrogeology in a hydrological basin. This basin is in an agricultural area typical of the Pampean region. We have collected a detailed record of precipitation, soil moisture and hydraulic head from stations designed for this purpose. The goal is to determine the recharge mechanisms of the aquifers of the Salto Chico, Hernandarias and Ubajay Formations in the Arroyo Palmar area. The available literature on the hydrogeology of these aquifers, and especially their recharge is very scarce. Auge and Santi papers are available, 2002; Auge et al., 2005; Santi 2000; Santi et al., 2009 and Filí et al., 1993 and seem to have significantly over estimated the actual recharge values by direct infiltration of rainfall. The Palmar creek watershed study area can be seen in Figure 1 (Machado et al., 2017). The Palmar creek basin has a young main channel in the low basin and is from young to mature in the middle and upper basin. This is a consequence of recent geological changes (Silva Busso and Machado, 2019). Land uses in the basin are agricultural and forestry. Much of the basin does not preserve the original biome, many species and natural ecosystems are preserved in El Palmar National Park (Silva Busso and Machado, 2019). An inescapable study on the recharge is that of Auge and Santi (2002) covering a very large area that that includes the study basin in this study. Auge and Santi (2002) used the weather data to propose that infiltration would reach $15 \%$ of the annual average precipitation considering it (without further analysis) to be a recharge of the free aquifer. We have calculated the recharge of aquifers from excess water in the ground using continuous registration sensors located at two control stations (Hernandarias He-01 and Ubajay Ub-01) located in the Palmar Basin (Fig. 4 and Table 1). We have carried out a detailed study of the soils and hydrogeology in the watershed of an agricultural area, characteristic of the Pampean region. We have collected a detailed record of precipitation, soil moisture and hydraulic loads from stations designed for this purpose.

\section{Results and Discussion}

Figures 5 and 6 show a summary of the characteristics of the soil on which the control stations and geology crossed by the observation piezometers have been located. The soils that develop in the area are two: In the Ubajay Formation entisols have specifically developed the Yuquerí series of the Oxyaquic Quartzipsamments family, aerobic with recent sandy, alluvial soil. In the Hernandarias Formation, richer in clays, vertisols of the San Guillermo Series belonging to the "fine, montmorillonitic, thermal" family of the Aristic Peludert that are characterized by the formation of retraction cracks in the dry season. This analysis will be used as a daily time scale. The period 11/9/2014 to $11 / 29 / 2015$ has been chosen as the basis for the analysis as it covers a complete hydrological cycle and presents continuity in the operation of all the sensors. Maximum, minimum and average values of soil humidity $\left(\theta_{(\mathrm{v} / \mathrm{v})}\right)$ and static levels are presented in Table 4 for station Ub-01 and Table 5 for the He-01 station. Figures 7 and 8 show the evolution of moisture in soil profiles and rainfall recorded during the study period. In Ub-01 there are drainage conditions that allow the transfer of surplus soil to the unsaturated area and aquifer. This station is representative of soils developed on the outcrops of the Ubajay Formation in the lower section of the Palmar basin, a forest area and with water exploitation use mainly for human use and livestock farming. At the He-01 station, the clay texture determines these abrupt moisture variations because in dry periods they contract and crack, accelerating drying and when it rains this facilitates the rapid ingress of water. Nevertheless, this effect is only seen on horizon l, the lower horizons maintain high humidity values and very little variables, reducing the infiltration of water surpluses. At the Ub-01 station (Fig. 9) there is a clear seasonality where the most effective rainfall is that which produces a measurable surplus. Recharge occurs at the end of winter and early spring. Figure 10 presents the data for station $\mathrm{He}-01$. In this case seasonality is only clearly observed in the deeper loggers. The first horizon has large oscillations, especially significant during the dry period. We have interpreted this as being the result of the existence of drying cracks that allow very quick access of water to the inside of the profile, even if the precipitation is very scarce. For its part, the application of the Reynolds and Elrick method, (1985), which evaluates the Ks of the whole of the soil column, offers values of 0.94 md-1 for Ub-01 and 0.0022 md-1 for the He-01, almost three orders of magnitude difference to Rosetta's estimates. 
The profiles of $\theta$ ry $\theta$ s and saturated hydraulic conductivity (table 8) show that both parameters are higher in the first $0.3 \mathrm{~m}$ of the profile (horizon I) falling steeply by almost an order of magnitude. The textural differences in depth decrease the secondary porosity and the permeability.

\section{Summary of Conclusions}

Studies so far available have considered the direct infiltration of precipitation water as the main recharge source of the Salto Chico aquifer. These studies considered that this recharge was between a maximum of $15 \%$ precipitation (Auge and Santi 2002; Auge et al.2005) and a minimum of 7\% (Santi 2002; Santi et al. 2009). Over the years, these values have been used as a reference for the management and planning of the available reserves of the aquifer resource. However, our results seem to show that vertical recharge is not the main water inlet into the aquifer and that previously estimated values significantly overestimate this component of the water balance. We have analysed the influence and magnitude of vertical recharge from a water balance in the unsaturated zone. The calculation of the net recharge was also included in the saturated zone. We have shown that the geological conditions of the basin influence the aquifer recharge. In the outcrop zone of the F. Ubajay the infiltration of surpluses allow a vertical transit equivalent to $6.61 \%$ with net recharge values of $1.41 \%$ precipitation. In the outcrop zone of $F$. Hernandarias the conditions do not favour the infiltration of water surpluses and only allow a transit equivalent to $0.89 \%$ with a net recharge of $0.04 \%$ precipitation. This scenario suggests that vertical recharge is not a dominant or quantitatively important process, which is atypical in Argentina's Pampean region. Therefore, vertical recharge is not a dominant or quantitatively important process in the recharge magnitudes of the Salto Chico aquifer. This scenario is atypical in relation to what happens in other areas of the Pampean region of the plain where vertical recharge is the most significant process in the net recharge of aquifers. Future research into this strategic environmental aspect with an impact on agricultural productivity should take a course consistent with the results we have shown. A new aquatic recharge model should be defined to be consistent with the measured data and a search for refill assessment and quantification methodologies different from those used so far should be carried out.

\section{Introducción}

\section{Objetivos}

Este trabajo tiene como objetivo determinar cuáles son los mecanismos de recarga de los acuíferos de las Formaciones Salto Chico, Hernandarias y Ubajay en la zona del Arroyo Palmar, provincia de Entre Ríos, Argentina. Se trata de un acuífero especialmen- te relevante en la economía regional pues es el más explotado, y conocer los mecanismos de recarga es fundamental para una óptima gestión del uso agrario del agua subterránea. Un segundo objetivo ha sido el cálculo de la tasa de recarga por infiltración directa de lluvia. La bibliografía disponible acerca del funcionamiento de estos acuíferos, y especialmente de su recarga directa por lluvia es muy escasa, apenas se disponen de cinco trabajos (Auge y Santi, 2002; Auge et al., 2005; Santi, 2002; Santi et al., 2009 y Filí et al., 1993) y como se verá más adelante los resultados cuantitativos manejados hasta este momento parecen sobreestimar notablemente los valores reales.

\section{Marco geográfico y socioeconómico}

La zona en estudio (Figura 1) se encuentra en un área de transición entre el clima subtropical y templado, sin estación seca o húmeda marcada. La temperatura media anual en la Estación Concordia ( $40 \mathrm{~km}$ al norte) es de $19^{\circ} \mathrm{C}$, oscilando entre 20 y $21^{\circ} \mathrm{C}$. Las precipitaciones anuales disminuyen en forma gradual de NE a SO variando entre los 1.000 y $1.300 \mathrm{~mm}$ (Aldonza, 2016). La época de mayores precipitaciones va de octubre a abril (73\% del total anual). En los meses de verano se observan situaciones de déficit hídrico (Rojas y Saluso, 1987).

Las determinaciones morfométricas de la cuenca hidrográfica del Arroyo Palmar (Machado et al., 2017) realizadas sobre la base del MDT-ALOS, muestran curvas hipsométricas entre los $10 \mathrm{~m}$ s.n.m. en la desembocadura y $70 \mathrm{~m}$ s.n.m. en las cabeceras, una altitud media de 55,00 m s.n.m. y una altitud más frecuente de 52,50 m s.n.m..

El área de cuenca es de $512,48 \mathrm{~km}^{2}$ y el perímetro es de $164,57 \mathrm{~km}$, La longitud del eje de cuenca es de $42,20 \mathrm{~km}$ y el ancho es de 12,14 km siendo su cauce principal de 24,55 km (Machado et al., 2017). El análisis porcentual de pendientes de la cuenca define aspectos fisiográficos que regulan en cierta medida la escorrentía no encauzada. La cuenca posee una pendiente media de $16,62 \%$, valor característico de relieves ondulados de pradera, y una pendiente media del cauce principal de $0,08 \%$ lo que indicaría velocidades de flujo encauzado relativamente bajas (Machado et al., 2017). Fisiográficamente la cuenca del Arroyo Palmar muestra un cauce principal joven en la cuenca baja y de joven a maduro en la cuenca media y alta, consecuencia de cambios geológicos recientes (Silva Busso y Machado, 2019).

Las actividades principales en la cuenca del Arroyo Palmar son de tipo agropecuarias, incluyen la ganadería extensiva, la silvicultura, citricultura, agricultura de secano de diferentes cereales y oleaginosas y agricultura de regadío de arroz. Subordinadamente se registra la cría avícola intensiva, las huertas y los viveros. Gran parte de la cuenca no conserva el bioma original, aunque muchas especies y ecosistemas naturales se conservan en el Parque Nacional El Pal- 

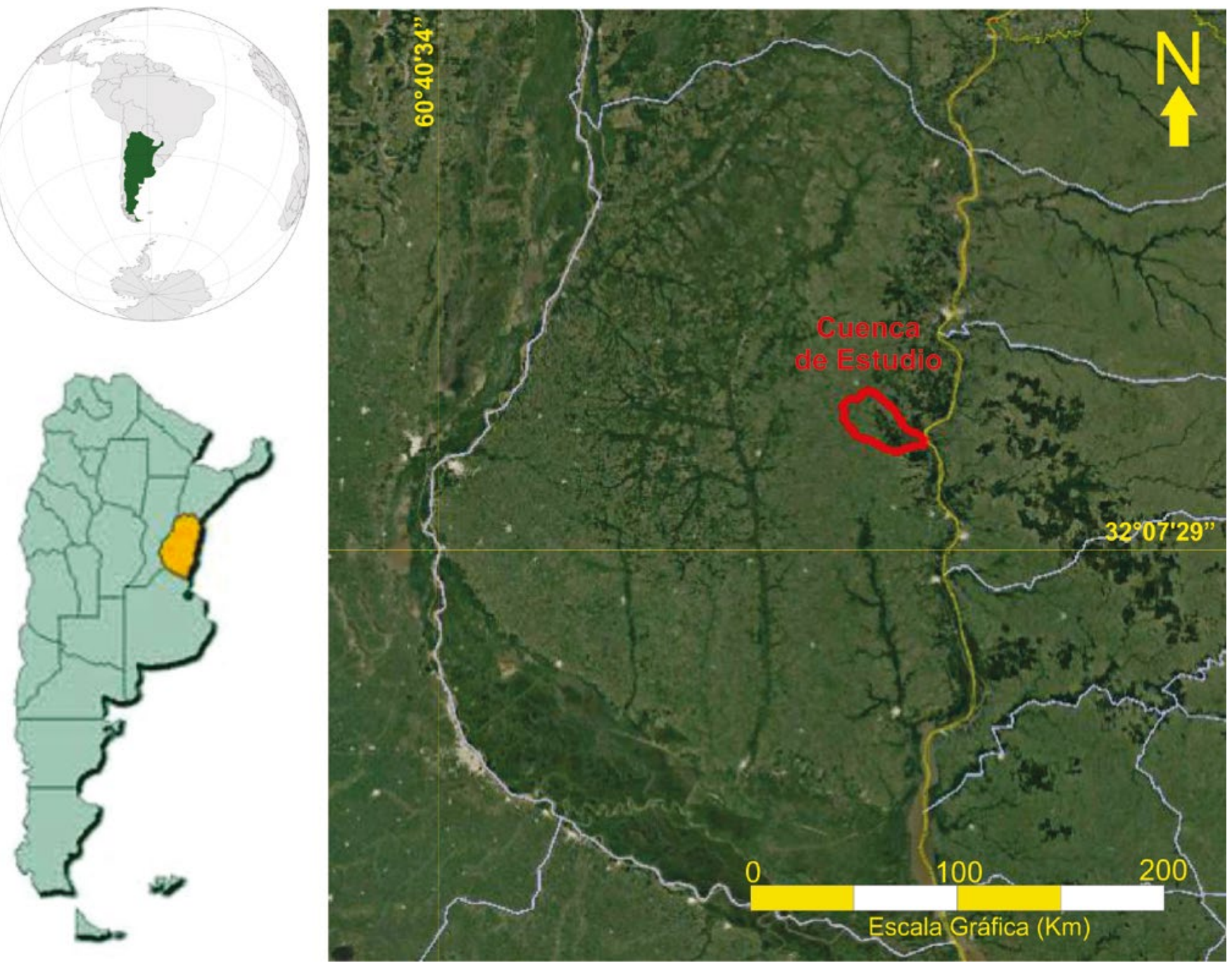

Figura 1. Ubicación de la Cuenca Arroyo Palmar, Entre Ríos, Argentina.

Figure 1. Location of the Palmar basin, Entre Ríos, Argentina.

mar que ocupa casi toda la cuenca baja (Silva Busso y Machado, 2019). A excepción del parque nacional, los usos agro-ganaderos cambian con el tiempo en función de los precios de mercado y la productividad, sobre todo la ganadería y el cultivo en secano.

El único estudio disponible sobre uso de suelo en la cuenca del Arroyo Palmar (Micou, 2003) define áreas de uso de suelo en la cuenca sobre la base de la interpretación de la imagen LANSAT TM y corroboración en campo. El estudio muestra que los usos más importantes son la agro-ganadería y la silvicultura (a pesar de que esta última comparte su espacio con el Palmar de la Reserva Nacional). Esta situación se mantiene a lo largo del tiempo hasta la actualidad. Según este autor, la Ruta Nacional N¹4 estaría actuando como una divisoria entre dos usos de suelo diferentes: hacia el este de tipo predominante forestal y hacia el oeste de tipo agrícola. Este cambio tan marcado del uso del suelo coincide en realidad con el límite del contacto geológico de las dos principales unidades aflorantes que conducen al desarrollo de di- ferentes tipos de suelo (Silva Busso y Machado, 2019).

Resulta así que la cuenca baja ubicada hacia oriente, además de contener la reserva natural el Palmar, mantiene cultivos de Eucalyptus grandis, Eucalyptus dunni, Pinus elliottii, Pinus taeda y Eucalyptus globulus, que resulta la actividad más significativa de esta zona. En la cuenca media y alta, hacia el poniente, la producción agrícola es la más significativa siendo el cultivo de arroz la más importante (Auge y Santi, 2002 y Auge et al., 2005). El arroz se riega a partir bombeo de agua subterránea explotando el Acuífero Salto Chico (Silva Busso y Amato, 2017). Se emplea fundamentalmente el riego a manta, manteniéndose el suelo inundado casi 40 días, lo que imposibilita la rotación de cultivos e induce una degradación más rápida del suelo. Además, según lo expuesto por Auge (2005) el método de riego es ineficiente y provoca una gran extracción de agua de los acuíferos calculando explotaciones entre $100-500 \mathrm{~m}^{3} / \mathrm{h}$ lo que, según dicho autor, implica la extracción de unos $360.000 \mathrm{~m}^{3}$ en cada temporada de riego. 


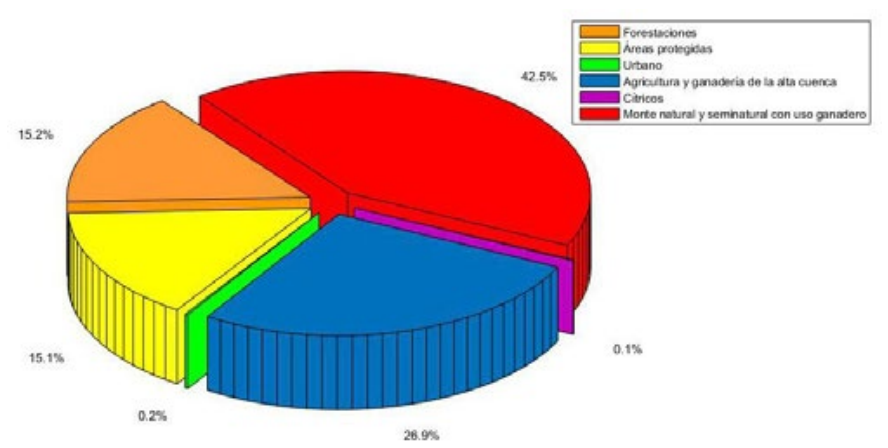

Figura 2. Usos de suelo en la Cuenca del Arroyo Palmar (Micou, 2003).

Figure 2. Land uses in the Palmar basin (Micou, 2003).

El esquema de uso del suelo descrito se replica en las cuencas adyacentes, que muestran características geológicas similares, razón que ayuda a entender la importancia de conocer los mecanismos de recarga de los acuíferos y su cuantificación. Esto último no solo posee importancia desde una visión productiva sino también de preservación del recurso, de las áreas protegidas y de su sostenibilidad.

\section{Marco geológico e hidrogeológico}

La geología e hidrogeología de interés en este estudio se desarrolla en las formaciones más modernas, algunas de las cuales contienen los principales acuíferos de la cuenca, y de gran parte de la región centro noroeste de la comarca entrerriana. La Formación Salto Chico (Gentile y Rimoldi, 1979) resulta en una sucesión de gravas silíceas que intercalan arenas cuarzosas estratificadas de granulometría media a gruesa sin faltar algún paquete de material limo arcilloso. La parte superior del conjunto afloraba al norte sobre el río Uruguay con colores ocre, rojizo y amarillento, hoy día cubierto por el espejo de agua de la presas Salto Grande, cruzando a la banda oriental del río. Presumiblemente y hacia el sur, esta unidad cruzaría nuevamente el Río Uruguay a la actual altura de la Ciudad de Colón ( $40 \mathrm{~km}$ al sur) siendo bisectada por los depósitos fluviales actuales del río Uruguay (Iriondo, 1996). Se le asigna edad Plio-pleistoceno (Gentile y Rimoldi, 1979) y se la correlaciona con el resto de las unidades de origen fluvial y edad comparable en el resto de la región pampeana y en la costa este de la República de Uruguay (Silva Busso y Amato, 2017). Los niveles suprayacentes a la F. Salto Chico son los que finalmente afloran toda la cuenca del arroyo Et Palmar, en Silva Busso y Machado, (2019) se definen y se cartografían estas unidades con más detalle. Las unidades aflorantes son dos; la F. Hernandarias y la F. Ubajay y ambas suprayacen a la F. Salto Chico. La Formación Hernandarias (Reig, 1956) es una cubierta sedimentaria de edad cuaternaria (Pleistocena) que se extiende sobre la mayor parte de la provincia de Entre Ríos. En la cartografía geológica (Bertolini, 1995), la F. Hernan- darias aparece dividida en cuatro grupos que son: a) Limos arcillosos con yeso macro y microscópico; b) arcillas limosas; c) Limos loessoides y d) limos areno arcillosos, cubriendo los cuatro grupos aflorantes gran parte de la superficie de la mencionada provincia Según Cordiní (1949), que realizó el estudio geológico de numerosas perforaciones en la provincia, observó que el espesor de la misma variaba entre 3 a 10 metros en el centro-norte de la provincia alcanzando los 15 metros en la zona norte donde se hallan los afloramientos costeros de la misma sobre el Río Paraná. La Formación Hernandarias se habría originado durante el Pleistoceno inferior a medio, durante el ciclo correspondiente a la primera glaciación considerado como el más largo durante el Cuaternario. Durante el ciclo siguiente (interglaciar) más húmedo hay predominio de la erosión, contribuyendo a la formación de la red de drenaje actual (Vitulich, 1982). Los estudios hidrogeológicos que han incluido evaluaciones de la recarga se han realizado en la región agro-ganadera donde aflora la F. Hernandarias pues en esta zona los suelos permiten el cultivo de arroz, principal demandante del recurso hídrico subterráneo del infrayacente Acuífero Salto Chico. La primera aproximación a este aspecto es de Filí et al (1993) que realizó un estudio en el centro y norte de la provincia de Entre Ríos (también área de afloramiento de la $F$, Hernandarias) y aporta dos conclusiones importantes. La primera es que, sobre la base de mediciones de humedad con sonda neutrónica, la F. Hernandarias posee poca humedad poral (aunque no aporta valores). La segunda es que, sobre la base de estudios isotópicos de $\mathrm{D}^{2} ; \mathrm{O}^{18}$ $\mathrm{y} \mathrm{H}^{3}$, las aguas del infrayacente Acuífero Salto Chico superan al menos los 30 años de edad.

Más recientemente (Auge y Santi 2002) estudian la recarga en un área que se extiende entre los $31^{\circ} 20^{\prime} y$ $32^{\circ} 40$ 'de latitud Sur. La cuenca estudiada se encuentra casi en el centro de esta región. Estos autores realizaron un balance usando el método de Thornthwaite y Mather (1957), que según dichos autores, ha brindado resultados aceptables en regiones con exceso hídrico. La escorrentía la calcularon sobre la base de escasos datos medidos en algunos arroyos de la región en periodos muy breves de tiempo. Usando los datos meteorológicos de esta amplia región, restando la evapotranspiración calculada y la escorrentía proponen que la infiltración alcanzaría el $15 \%$ de la precipitación media anual considerándola, sin más análisis, como recarga del acuífero libre. Se alude también a que el contenido de arcillas en la F. Hernandarias provoca en periodos secos grietas que aumentan la infiltración. También mencionan que la presencia dominante de bicarbonatos en las aguas del acuífero como un indicador claro del proceso de infiltración vertical. No obstante, reconocen que no tienen datos de infiltración, pero calculan la recarga considerando el valor del $15 \%$ (Auge y Santi, 2002 y Auge et al., 2005). Para el sudeste de Entre Ríos (Santi 2002, Santi et al. 2009) proponen que la recarga del acuífero se produce por infiltración 
vertical directa a través de los limos arcillosos de la Formación Hernandarias y el balance hídrico, calculado de forma similar al anterior, da un valor para la recarga del acuífero del 7\% de la precipitación media anual.

La Formación Ubajay (Gentile y Rimoldi, 1979).ubicada en la margen derecha del Río Uruguay se extiende ente la desembocadura del Río Mocoretá y la ciudad de Concepción de Uruguay y las cotas altimétricas del techo coinciden en gran medida con la topografía del terreno. Está compuesta de arenas finas a medianas de colores rojos, amarillentos y ocres donde intercalan niveles conglomeráticos matriz sostén de poco espesor con rodados de ópalo, calcedonia, cuarzo blanco y eventualmente fragmentos de basalto. También se observan intercalaciones de arcillas y limos de los colores mencionados observables en la secuencia vertical formando pequeños bancos. Suelen ser sedimentos sin consolidar que raramente supera los 15 metros de espesor y hacia el techo se observan con frecuencia niveles silcretizados muy consolidados raramente de más de un metro de espesor. Posteriormente, ha sido estudiada en Entre Ríos por Iriondo (1980), Silva Busso y Amato, (2013) y Silva Busso y Amato, (2017) y en la cuenca del Arroyo Palmar se ha descripto con más detalle en Silva Busso y Machado, (2019). La misma sería de edad Holocena y lateralmente hacia el oeste desaparece del registro y comienzan los afloramientos de la F. Hernandarias con la que tendría un contacto erosivo. Desde un punto de vista hidrogeológico, los primeros estudios de esta unidad los realiza Masú et al., (2011), justamente en la cuenca bajo de estudio, mencionando que el limo-arcilla tiene una notoria influencia en su productividad acuífera limitando su aprovechamiento y explotación.

\section{Metodología}

Para el cálculo de la recarga de los acuíferos se ha partido del cálculo del exceso de agua en el suelo mediante sensores de registro continuo ubicados en dos estaciones de control (Hernandarias He-01 y Ubajay Ub-01) que se ubican en la Cuenca del Arroyo Palmar (Fig. 3 y Tabla 1), un afluente del Río Uruguay en el Departamento de Colón, Provincia de Entre Ríos, Argentina (Fig. 1). Además del control de humedad, se midieron los niveles estáticos del agua subterránea y se recolectaron datos meteorológicos tal como se describe más adelante.

\begin{tabular}{|c|c|c|c|c|}
\hline Estación & Latitud (S) & $\begin{array}{c}\text { Longitud } \\
\text { (O) }\end{array}$ & $\begin{array}{c}\text { Cota (m } \\
\text { s.n.m.) }\end{array}$ & $\begin{array}{c}\text { Forma- } \\
\text { ción }\end{array}$ \\
\hline He-01 & $31^{\circ} 41^{\prime} 34,6^{\prime \prime}$ & $58^{\circ} 26^{\prime} 57,7^{\prime \prime}$ & 61,70 & $\begin{array}{c}\text { Hernan- } \\
\text { darias }\end{array}$ \\
\hline Ub-01 & $31^{\circ} 52^{\prime} 55,9^{\prime \prime}$ & $58^{\circ} 19^{\prime} 13,2^{\prime \prime}$ & 28,51 & Ubajay \\
\hline
\end{tabular}

Tabla 1. Resumen de las características de las estaciones hidrogeológicas.

Table 1. Summary of the hydrogeological station characteristics.
Se escogió la cuenca del Arroyo Palmar debido a la diversidad de usos de suelo y del agua subterránea y a la existencia de un área protegida (parque del Palmar), además en la zona se definen dos áreas bien delimitadas de afloramiento de las F. Hernandarias y la F. Ubajay que en conjunto cubren más del $95 \%$ de la superficie de la cuenca. Sobre la costa del Río Uruguay en la desembocadura del Arroyo Palmar, aflora también la F. Puerto Yerúa cuya superficie es despreciable en comparación a las formaciones mencionadas, así que son las primeras las que condicionan la génesis de suelos y sobre todo las propiedades hidráulicas de los materiales aflorantes. Cada estación de control cuenta con tres registradores de humedad y temperatura de suelo, ubicados a las profundidades en las que se identificaron cambios edáficos en calicatas para tal fin. El equipamiento también incluye un termómetro de aire en superficie (Tabla 2) y a pocos metros cada instalación se encuentra un piezómetro de observación que cuentan con un registrador de presión que mide la carga hidráulica. El resumen de los diseños de los piezómetros puede verse en la Tabla 3.

\begin{tabular}{|l|l|l|l|l|l|l|}
\hline Estación & $\begin{array}{c}\text { Forma- } \\
\text { ción }\end{array}$ & $\begin{array}{c}\text { Orden } \\
\text { de suelo }\end{array}$ & $\begin{array}{l}\text { TDR- } \\
\mathbf{3}(\mathbf{m})\end{array}$ & $\begin{array}{l}\text { TDR- } \\
\mathbf{2}(\mathbf{m})\end{array}$ & $\begin{array}{l}\text { TDR- } \\
\mathbf{1}(\mathbf{m})\end{array}$ & $\begin{array}{c}\text { RT-1 } \\
(\mathbf{m})\end{array}$ \\
\hline He-01 & $\begin{array}{l}\text { Hernan- } \\
\text { darias }\end{array}$ & Entisol & 0,3 & 0,6 & 1,2 & $+0,1$ \\
\hline Ub- 01 & Ubajay & Vertisol & 0,3 & 0,7 & 1,5 & $+0,1$ \\
\hline
\end{tabular}

Tabla 2: Ubicación de los sensores de humedad del suelo. Table 2: Location of the soil moisture sensors.

\begin{tabular}{|l|c|c|c|c|c|}
\hline Estación & Formación & $\begin{array}{c}\text { Tramo } \\
\text { acuífe- } \\
\text { ro (m) }\end{array}$ & $\begin{array}{c}\text { Filtros } \\
\text { (m) }\end{array}$ & $\begin{array}{c}\text { Ce- } \\
\text { menta- } \\
\text { do (m) }\end{array}$ & $\begin{array}{c}\text { HOBO } \\
\text { (m) }\end{array}$ \\
\hline He-01 & $\begin{array}{c}\text { Salto Chi- } \\
\text { co }\end{array}$ & $\begin{array}{c}30-52 \text { y } \\
61-92\end{array}$ & $\begin{array}{c}35-2 \text { y } \\
62-92\end{array}$ & $0-26$ & 5 \\
\hline Ub- 01 & Ubajay & $0-18$ & $6-15$ & $0-6$ & 34 \\
\hline
\end{tabular}

Tabla 3. Características los piezómetros de observación en metros desde la superficie.

Table 3. Characteristics of the observation piezometers (metres from the surface).

El sondeo de la estación He-01 capta el acuífero superior (Salto Chico), la F. Hernandarias suprayacente es un acuícludo. El sondeo de la estación Ub-01 capta el Acuífero Ubajay, se trata de un acuífero libre de baja permeabilidad (Silva Busso y Amato 2017) bajo el cual se dispone el Acuífero Salto Chico con quien está conectado verticalmente.

El instrumental de medición de cada estación se compone de tres sensores 5TM (TDR- time domain reflectometry) marca Decagon Devices que miden la temperatura y el contenido volumétrico de agua en el suelo con una exactitud de $+/-2 \%$. Además, se cuenta con un cuarto sensor el RT-1, también de la misma marca que registra la temperatura ambiente a $0,1 \mathrm{~m}$ so- 
bre el nivel del suelo. Los datos obtenidos se almacenan en un registrador EM50 del mismo fabricante que cuenta con 5 canales de medición con una capacidad de registro de 36.000 datos y una autonomía entre 8 y 12 meses. El nivel piezométrico se registra en un sensor HOBO U20L-01, este dispositivo tiene un error de +/$0,6 \mathrm{~cm}$ y registra de forma simultánea la temperatura con una precisión de $+/-0,44^{\circ} \mathrm{C}$. La estación meteorológica (Palmar-01 PM-01) es del modelo Capbell Scientific CR1000, se encuentra dentro de las dependencias del parque nacional en la cuenca baja en $31^{\circ} 51^{\prime} 50,28^{\prime \prime} S$ y $58^{\circ} 15^{\prime} 34,21^{\prime \prime} \mathrm{O}$, a cota $30,18 \mathrm{~m}$ s.n.m.

La estación registró la temperatura del aire, mínima y máxima, radiación incidente, radiación reflejada y precipitación. Los datos se toman cada hora y están sincronizados entre sí. De los cinco años con datos disponibles se ha seleccionado el periodo entre el 9/11/2014 y $29 / 11 / 2015$ en el que las cuatro estaciones han funcionado de forma continua e incluye un ciclo hidrológico completo.

El agua infiltrada cada día $\left(I_{n}\right)$ corresponde al exceso del agua ingresada sobre el valor dela capacidad de campo multiplicado por el espesor del horizonte considerado (Eq. 1).

Esta agua se acumula al flujo precedente (Eq. 2), hasta que supera la capacidad de campo y percola. Cuando el agua atraviesa el horizonte situado bajo la zona radicular constituye la recarga del acuífero $\left(\mathrm{I}_{\mathrm{t}}\right)$.

$I_{n}=\left(\theta_{n}-\theta_{c c}\right) \times b_{n}$

Eq. 1

Si $I_{n}<0$ entonces

$I_{t}=\sum^{n}{ }_{l n<0} I_{n}$

Eq. 2

Donde

$I_{n}=$ Excedente gravífica en cada paso de tiempo $(\mathrm{mm})$

$\theta_{n}=$ Humedad de suelo en cada paso de tiempo $(v / v)$

$\theta_{c c}=$ Capacidad de campo $(v / v)$

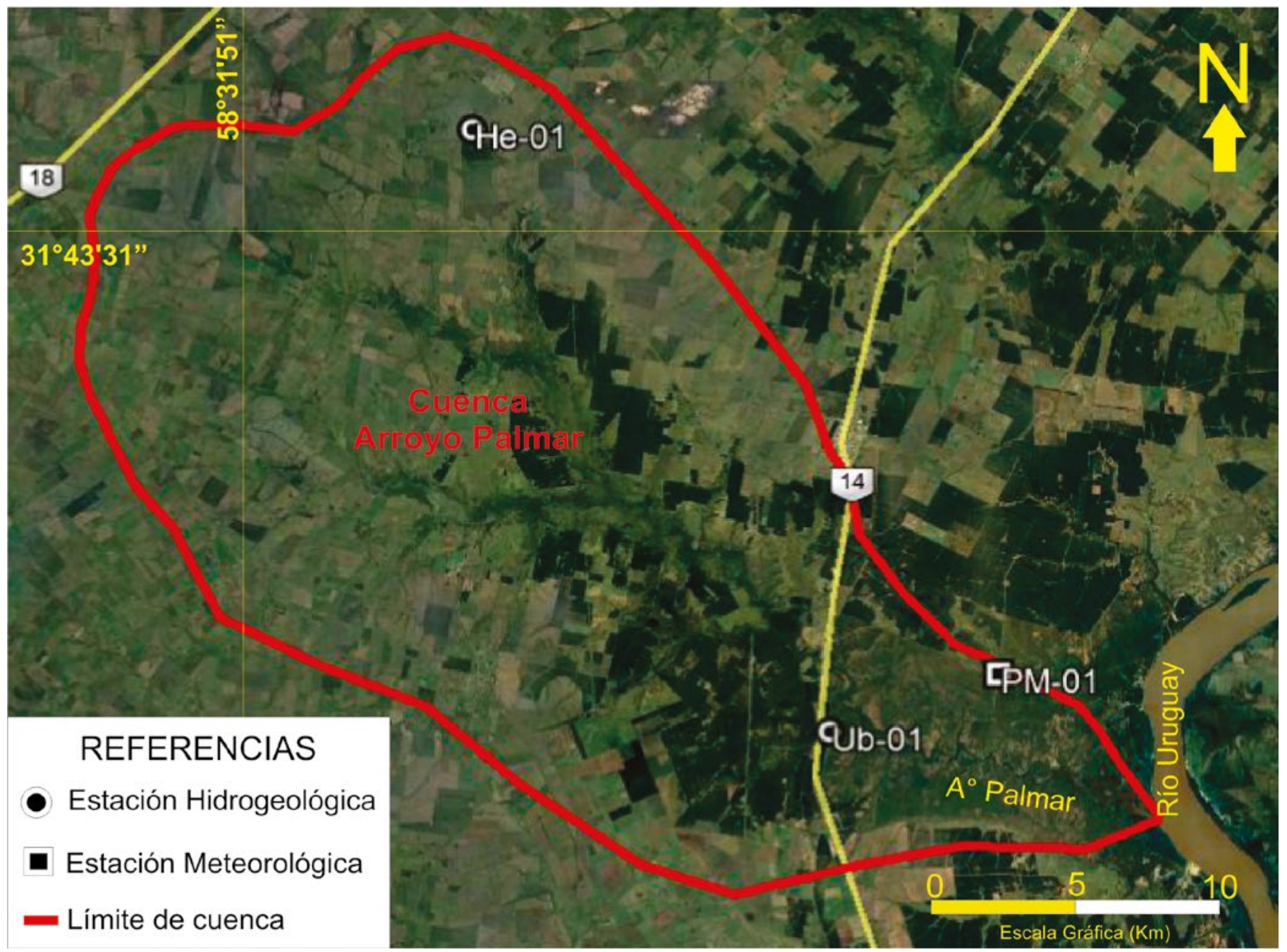

Figura 3. Ubicación las estaciones de registro en la Cuenca Arroyo Palmar. Figure 3. Location of the registration stations in Palmar Basin. 
$b_{n}=$ Espesor de la capa de suelo $(\mathrm{mm})$

$I_{t}=$ Excedente total de cada capa que puede moverse por gravedad $(\mathrm{mm})$

Los datos para la caracterización física del suelo se han obtenido directamente mediante procesos estándar o simulación. La capacidad de campo $\theta_{\text {cc }}$ del horizonte I se determinó en laboratorio por el método de Richards y Weaver (1944). En el resto de los horizontes se empleó el método de Maldonado (2001), en base a los datos de medidas de humedad en continuo determinados en campo. El análisis textural se realizó en el laboratorio de sedimentología del Dpto. de Geología de la Universidad Buenos Aires mediante la técnica tamizado y serigrafía. La permeabilidad hidráulica saturada y los parámetros de retención del suelo se han obtenido mediante la aplicación del programa Rosetta (Schaap et.al., 1998) que se basa en la formulación desarrollada por van Genuchten (1980) y Mualem (1976).

La recarga neta se ha calculado mediante la metodología propuesta por Lerner (1990) se trata de un método de balance directo que el autor denomina recarga neta medida por método piezométrico. Para aplicar este método se emplean las ecuaciones (3) y (4).

$\begin{array}{ll}\Delta_{N e}=\sum_{n=1}\left(N e_{n}-N e_{n-1}\right) & \text { Eq.3 } \\ R_{n}=\left(\Delta_{N e}\right) \times s & \text { Eq. } 4 \\ \text { Donde } & \end{array}$

$\Delta_{\mathrm{Ne}}=$ Variación del nivel estático $(\mathrm{mm})$ entre dos periodos de tiempo ( $n$ : final y $n-1$ : inicial)

$\mathrm{s}=$ Porosidad específica o coeficiente de almacenamiento (adimensional)

\section{$\mathrm{R}_{\mathrm{n}}=$ Recarga neta $(\mathrm{mm})$}

Este método presenta algunas limitaciones (Healy y Cook 2002): a) no puede emplearse si no hay oscilación del nivel piezométrico (régimen transitorio) los niveles oscilan en ambos acuíferos (ver figuras 11 y 12), aunque estas oscilaciones son realmente pequeñas, en el caso de la estación de Hernandarias de apenas $80 \mathrm{~cm}$ todo el periodo estudiado y en Ubajay de 1,1 metros. b) Las estaciones deben ser representativas del área de recarga. c) Proporciona mejores resultados si se aplica para niveles piezométricos someros, como sucede en los acuíferos estudiados. El valor del coeficiente de almacenamiento también es un factor limitante debido a la heterogeneidad del acuífero, se trata además de la variable más sensible en el cálculo. Los valores de coeficiente de almacenamiento empleados son los obtenidos por Masú et al., (2011), Silva Busso y Amato (2017) y Silva Busso y Machado (2019) para el acuífero en la cuenca del Arroyo Palmar. Identificar la causa de las fluctuaciones del nivel piezométrico es un aspecto complejo pues dependen también de numerosos factores de muy difícil control, como la existencia de bombeos o la relación río-acuífero.

\section{Resultados y discusión}

\section{Caracterización geológica e hidrogeológica}

En las Figuras 4 y 5 se muestra un resumen de las características de los suelos sobre los que se han ubicado las estaciones de control y de la geología atravesada por los piezómetros de observación. En la zona en estudio (INTA, 1990) se desarrollan dos tipos de suelo según el criterio taxonómico del Soil Survey Staff (1975) sobre la F. Ubajay predominantemente Entisoles, suelos muy poco evolucionados sin desarrollo de horizontes diagnóstico y compuesto principalmente por material regolítico edáficamente inalterado; sobre la $\mathrm{F}$, Hernandarias, mucho más rica en arcillas se desarrollan Vertisoles que se caracterizan por la formación de profundas grietas de retracción en la época más seca del año. En base a los suelos dominantes ya es de prever un comportamiento hidráulico netamente diferente en los suelos descritos sobre cada una de las formaciones.

En la Figura 4 se muestra la descripción del piezómetro situado en la estación Ub-01. Su profundidad total es de 40 metros, desde allí hasta los $30 \mathrm{~m}$ b.b.p. (metros bajo boca de pozo) atravesó parcialmente la F. Puerto Yeruá representada aquí por un paquete de arenas finas y limos de color blanquecino (2,5Y 8/2) moderadamente silicificados que desarrolla un acuífero fisurado de baja permeabilidad.

Entre los 18 - $30 \mathrm{~m}$ b.b.p. se observa un paquete fundamentalmente de gravas de color castaño-amarillento (10YR 8/8) con individuos de composición silícea-calcedonia, clasto-sostén, poco consolidado atribuible a la F. Salto Chico que constituye el acuífero regional más importante. Finalmente, entre los $0-18$ $m$ b.b.p. se observa la unidad más somera la $F$. Ubajay que constituye un complejo y poco seleccionado depósito cuya sección basal es una arcilla verde clara (5Y 8/3) seguida por arenas gravas, gravas matriz-sostén (con elevado contenido de finos) rematando la secuencia con limos arenosos de colores ocres (5YR $8 / 8$ ). Esta unidad es litológicamente muy variable en la cuenca del Arroyo Palmar predominando sedimentos psamíticos. La misma presenta moderada a baja permeabilidad con acuíferos que tienden a ser libres (a veces semi-libres), con las salinidades bajas (menores a $300 \mathrm{mg} / \mathrm{l}$ ) y en ocasiones con una tendencia a comportarse como acuitardo (Silva Busso y Amato, 2017). El nivel estático medido en el momento de la construcción del piezómetro e instalación de los registradores fue de 2,4 m b.b.p..

Los suelos desarrollados a partir de esta unidad lo hacen sobre arenas re-transportadas y depositadas de origen fluvial, son mal drenados y con capa freática poco profunda. El perfil edáfico se realizó a partir de una calicata de 2 metros de profundidad. 


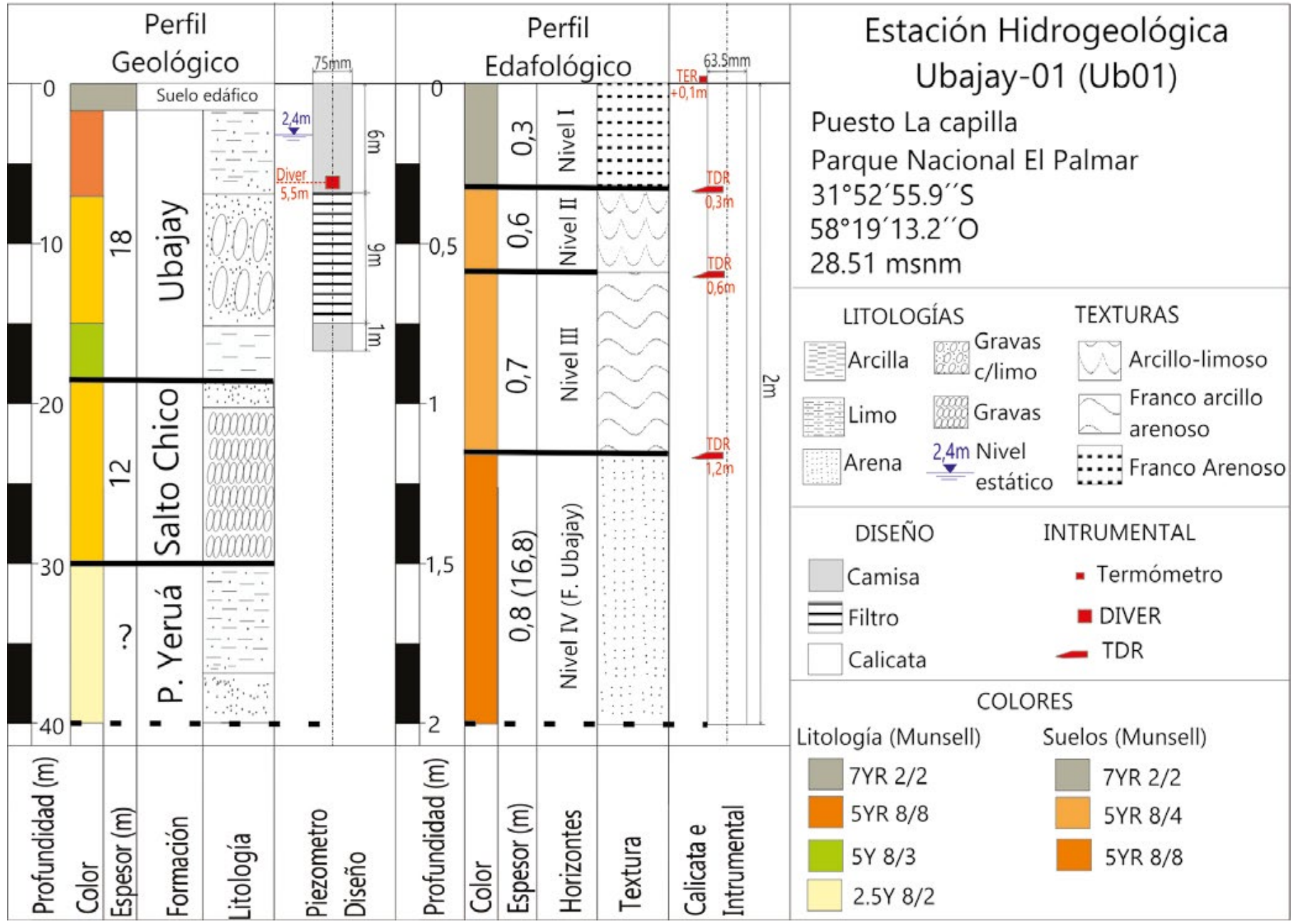

Figura 4. Caracterización de la estación hidrogeológica Ubajay-01.

Figure 4. Characterization of the Ubajay-01 hydrogeological station.

El primer horizonte (I) de suelo se desarrolla ente los $0-0,3$ m b.b.p. de color 7YR 2/2 (castaño grisáceo) in situ en húmedo; texturalmente franco arenoso (Arena: 60\%; Limo: 30\%; Arcilla: 10\%) y de estructura granular sin consolidar. Se observan niveles ferruginosos con cierto bandeado y transición inferior difusa o gradual.

El segundo horizonte (II) está ubicado entre los 0,3$0,6 \mathrm{~m}$ b.b.p. presenta color 5 YR $8 / 4$ (castaño rojizo claro) in situ en húmedo y se caracteriza por su textura arciIlo-limosa (Arena: 12\%; Limo: 48\%; Arcilla: 40\%).Se observa una estructura masiva, sin consolidar y plástica con manchas ferruginosas y de transición inferior neta.

El tercer horizonte (III) está entre los 0,6 - 1,2 m b.b.p., presenta color 5 YR $8 / 8$ (castaño rojizo u ocre) in situ en húmedo y se caracteriza por su textura Franco arcillo-arenosa (Arena: 52\%; Limo: 27\%; Arcilla: 21\%). Nuevamente, este horizonte es de estructura granular, sin consolidar y transición inferior difusa con el cuarto horizonte (IV) que constituye la formación parental. Se ha medido in situ la conductividad hidráulica empleando la propia calicata y aplicando el mé- todo de Reynolds y Elrick, (1985) que mantiene una carga hidráulica constante arrojando un resultado de $0,94 \mathrm{~m} / \mathrm{d}$, dato que puede considerarse representativo de todo el perfil edáfico.

Según la carta de suelos escala 1:50.000 del departamento de Colón (INTA, 2002), se trata de un suelo comparable a los de la Serie Yuquerí perteneciente a la familia "no ácida, térmica" de los Cuarzisamentes óxicos ácuicos (suelo aluvial reciente, arenoso).

En la Figura 5 se resumen las características del suelo y formación acuífera en la estación He-01. El piezómetro de estudio alcanzó los 94 metros de profundidad desde allí hasta los $92 \mathrm{~m}$ b.b.p. se atravesó el techo de la F. Paraná representada aquí por un paquete de arcillas de color verde-azuladas (5BG 3/6), cohesivas, plásticas de muy baja permeabilidad y que constituyen un acuícludo regional muy conspicuo. Entre los 26 - $92 \mathrm{~m}$ b.b.p. se observa un paquete mayormente gravoso de color castaño-amarillento (10YR 8/8) con individuos de composición silícea-calcedonia, clasto-sostén y poco consolidado que es nuevamente atribuible a la F. Salto Chico. Aunque en este perfil es importante destacar la 
potencia $(66 \mathrm{~m})$ relacionado con un control estructural norte-sur que profundiza a geología previa además de la probable presencia de dos ciclos fluviales en litofacies que típicamente se asocian con paleocanales (Silva Busso y Amato, 2017).

Esta litología y arquitectura sedimentaria explican las excelentes capacidades productivas del acuífero que lo convierten en el más importante de la provincia de Entre Ríos. En el tope de la secuencia, entre los $0-26 \mathrm{~m}$ b.b.p., la unidad más somera y aflorante es la F. Hernandarias. Aquí se compone de una arcilla de color gris plomizo a verdoso que alcanza un espesor importante $(26 \mathrm{~m})$, con finas láminas de limos donde se observan intercalaciones de carbonato de calcio formando rodados, concreciones de pocos milímetros y agregados pulverulentos manchados por óxido de manganeso.
No se descarta la presencia de yeso aunque no fue observado in situ. Hacia el techo de la secuencia resulta más limo-arcillosa, no obstante la arcilla resulta muy plástica y cohesiva. En acuerdo con Vitulich (1982) se asume la probable composición montmoriIlonítica de las arcillas. En la cuenca del arroyo Palmar esta formación sólo está ausente en los valles de los arroyos donde la erosión fluvial del cauce está aproximadamente por debajo de la cota $40 \mathrm{~m}$ s.n.m., pero en la zona de la estación He-01 es la única unidad aflorante, incluso observable en el fondo de los cauces fluviales. El nivel estático del primer acuífero (F. Salto Chico) se situaba en el momento de la instalación de los registradores a $28,8 \mathrm{~m}$ b.b.p.. El suelo de esta unidad está desarrollado sobre un depósito lacustre somero y/o palustre de clima seco, precipitación media

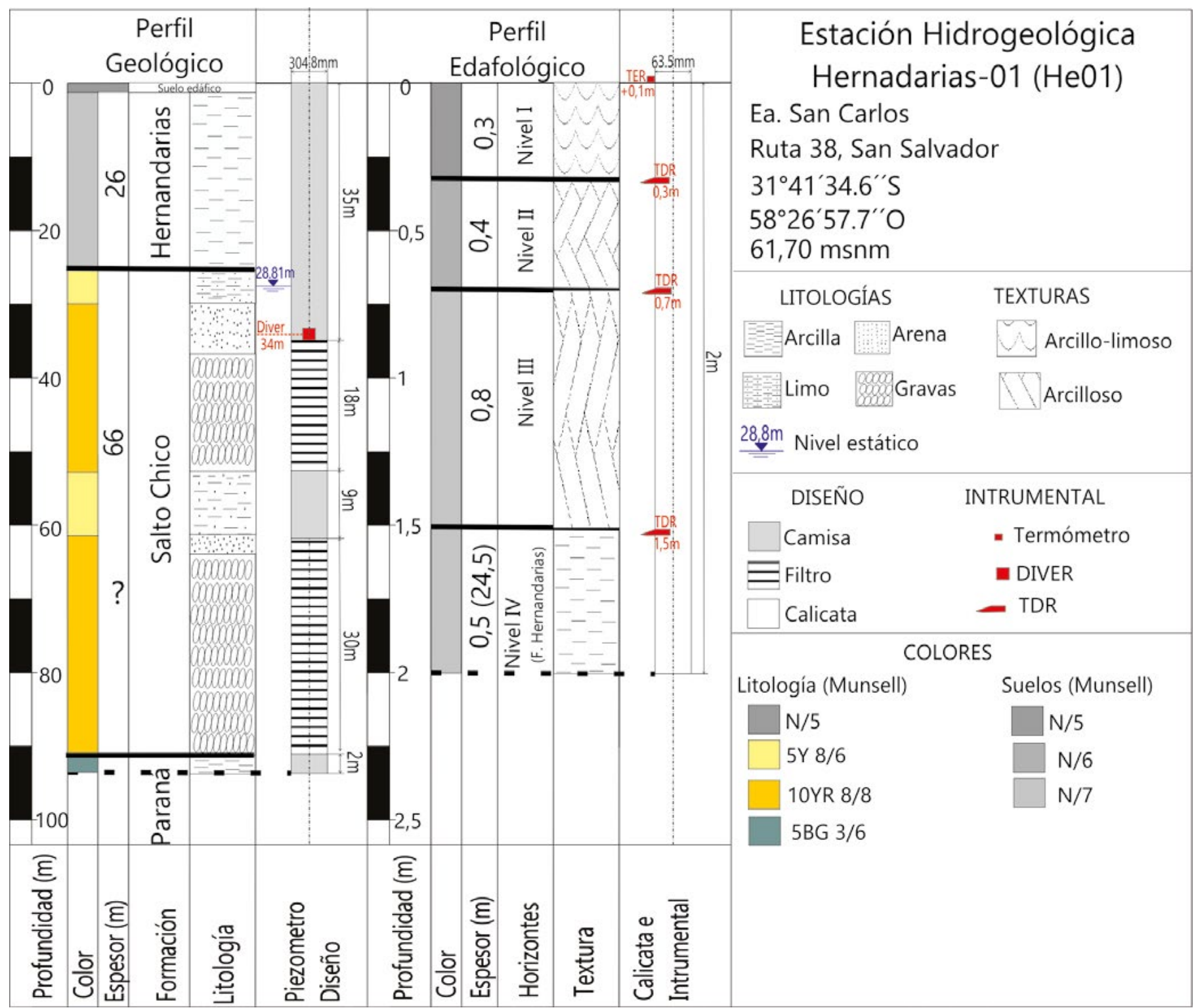

Figura 5. Caracterización de la estación hidrogeológica Hernandarias-01.

Figure 5. Characterization of the Hernandarias-01 Hydrogeological Station. 
en torno a 1000-1200 mm/año regularmente drenado; de escurrimiento superficial medio y capa freática ausente en su formación parental.

El primer horizonte (I) se desarrolla ente los $0-0,3$ $\mathrm{m}$ b.b.p., de color $\mathrm{N} / 5$ in situ en húmedo y texturalmente arcillo limoso (Arena: 14,6\%; Limo: 45,2\%; Arcilla: $40 \%$ ). Presenta estructura en bloques subangulares de tamaño medio, friable en húmedo, con escasos barnices y transición inferior difusa o gradual. El segundo horizonte (II) está ubicado entre los 0,3 $0,7 \mathrm{~m}$ b.b.p. presenta color $\mathrm{N} / 6$ in situ en húmedo y se caracteriza por su textura arcillosa (Arena: 12,5\%; Limo: 40\%; Arcilla: 47,5\%). Se observa una estructura en prismas irregulares y pequeños bloques angulares, es friable y posee abundantes barnices. Se detectan concreciones carbonáticas y ferruginosas con una transición inferior gradual. El tercer horizonte (III) se lo identifica entre los 0,7- 1,5 m b.b.p., de color N/7 in situ en húmedo se caracteriza por su textura arcillosa (Arena: 6,5\%; Limo: 34,5\%; Arcilla: 59\%). Posee una estructura masiva, friable, cohesiva con mayor presencia de concreciones calcáreas y transición inferior difusa con el cuarto horizonte (IV) que constituye la formación parental. La conductividad hidráulica, medida sobre la propia calicata (Reynolds y Elrick1985) se sitúa en $0,0022 \mathrm{~m} / \mathrm{d}$. Según la carta de suelos escala 1:50.000 del departamento de San Salvador (INTA, 1999) se trata de un suelo de la Serie San Guillermo perteneciente a la familia "fina, montmorillonítica, térmica" de los Peludertes árgicos.

\section{Análisis de los datos de las estaciones hidrogeológicas}

Los registradores de campo han tomado datos con frecuencia horaria, aunque en este análisis se va a emplear como escala temporal de análisis el día. Se ha elegido el periodo 9/11/2014 al 29/11/2015 como base para el análisis pues abarca un ciclo hidrológico completo y presenta continuidad en el funcionamiento de todos los sensores. La estación meteorológica PM-01, representativa de ambas estaciones, totaliza en el periodo de estudio una precipitación acumulada de $1473,7 \mathrm{~mm}$ con un máximo de precipitaciones diarias de $125,0 \mathrm{~mm}$ y un promedio de $3,8 \mathrm{~mm}$. Climáticamente el año en estudio es algo atípico, las precipitaciones de episodio de tormenta son más copiosas de lo habitual y se observa cierta estacionalidad, con un periodo más húmedo en primavera-verano y más seco en otoño-invierno, no obstante los máximos se sitúan dentro de los episodios de tormenta torrencial de la región (Aldonza, 2016).

Se presentan en la Tabla 4 para la estación Ub-01 y en la Tabla 5 para la estación He-01 los valores máximos, mínimos y promedio de la humedad de suelo $\left(\theta_{(\mathrm{v} / \mathrm{v})}\right)$ y de los niveles estáticos. La amplitud de oscilación registrada en los niveles fue de $0,85 \mathrm{~m}$ para la UB-01 y $0,87 \mathrm{~m}$ para la $\mathrm{He}-01$.

\begin{tabular}{|l|r|r|r|r|r|}
\hline & $\begin{array}{c}\text { TDR-3 } \\
\theta_{(v / v)}\end{array}$ & $\begin{array}{c}\text { TDR -2 } \\
\theta_{(v / v)}\end{array}$ & $\begin{array}{c}\text { TDR-1 } \\
\theta_{(v / v)}\end{array}$ & $\begin{array}{c}\text { Ne (m } \\
\text { b.b.p.) }\end{array}$ & $\begin{array}{c}\text { Pz (m } \\
\text { s.n.m.) }\end{array}$ \\
\hline Max & 0,29 & 0,37 & 0,36 & 2,53 & 26,79 \\
\hline Min & 0,02 & 0,02 & 0,03 & 3,38 & 27,64 \\
\hline Prom. & 0,10 & 0,19 & 0,19 & 2,92 & 27,25 \\
\hline
\end{tabular}

Tabla 4. Humedad del suelo y nivel piezométrico en la estación Ub01(9/11/2014 a 29/11/2015).

Table 4. Soil moisture and piezometric level at station Ub-01 (from 9/11/2014 to $29 / 11 / 2015)$.

\begin{tabular}{|l|r|r|r|r|r|}
\hline & $\begin{array}{c}\text { TDR-3 } \\
\theta_{(v / v)}\end{array}$ & $\begin{array}{c}\text { TDR -2 } \\
\theta_{(v / v)}\end{array}$ & $\begin{array}{c}\text { TDR-1 } \\
\theta_{(v / v)}\end{array}$ & $\begin{array}{c}\text { Ne (m } \\
\text { b.b.p.) }\end{array}$ & $\begin{array}{c}\text { Pz (m } \\
\text { s.n.m.) }\end{array}$ \\
\hline Max & 0,74 & 0,75 & 0,61 & 29,19 & 31,64 \\
\hline Min & 0,11 & 0,14 & 0,17 & 30,06 & 32,51 \\
\hline Prom. & 0,48 & 0,61 & 0,54 & 29,63 & 32,07 \\
\hline
\end{tabular}

Tabla 5. Humedad del suelo y nivel piezométrico en la estación He01 (9/11/2014 a 29/11/2015).

Table 5. Soil moisture and piezometric level at station He-01 (from 9/11/2014 to $29 / 11 / 2015$ ).

Las Figuras 6 y 7 muestran la evolución de la humedad de los perfiles de suelo y las precipitaciones registradas en el periodo de estudio. Como se puede observar (Figura 6), en la estación Ub-01 el desfase entre las precipitaciones y el cambio de humedad en los horizontes de suelo I y II es mínimo, de pocas horas a un día. El horizonte III puede demorar un poco más de un día. Los valores de humedad muestran caídas significativas durante el periodo seco de otoño e invierno, especialmente en los horizontes de textura más arenosa. Parece que en esta estación existen condiciones de drenaje que permiten la transferencia de excedentes del suelo a la zona no saturada y al acuífero. Esta estación es representativa de suelos desarrollados sobre los afloramientos de la F. Ubajay en la sección inferior de cuenca del Arroyo Palmar, una zona forestal y con explotación del agua para uso humano y ganadero principalmente. La Estación He-01 (Figura 7) presenta un registro diferente. El horizonte I se observa una correlación clara entre los valores de humedad y las precipitaciones, con escaso o nulo desfase, sin embargo, los registros de los horizontes II y III apenas muestran variación, y no correlaciona con el momento en que precipita localmente. La influencia de los periodos secos en otoño e invierno solo son observables en el horizonte I mientras que los horizontes II y III apenas muestran variación en los diferentes periodos pluviométricos. La textura arcillosa del horizonte I determina esas bruscas variaciones de humedad, en periodos secos se contraen y agrietan acelerando la desecación pero cuando llueve esas mismas grietas favorecen el ingreso rápido del agua. Este efecto solo se observa en el horizonte I. Los horizontes inferiores mantienen valores de humedad elevados y muy estables. 

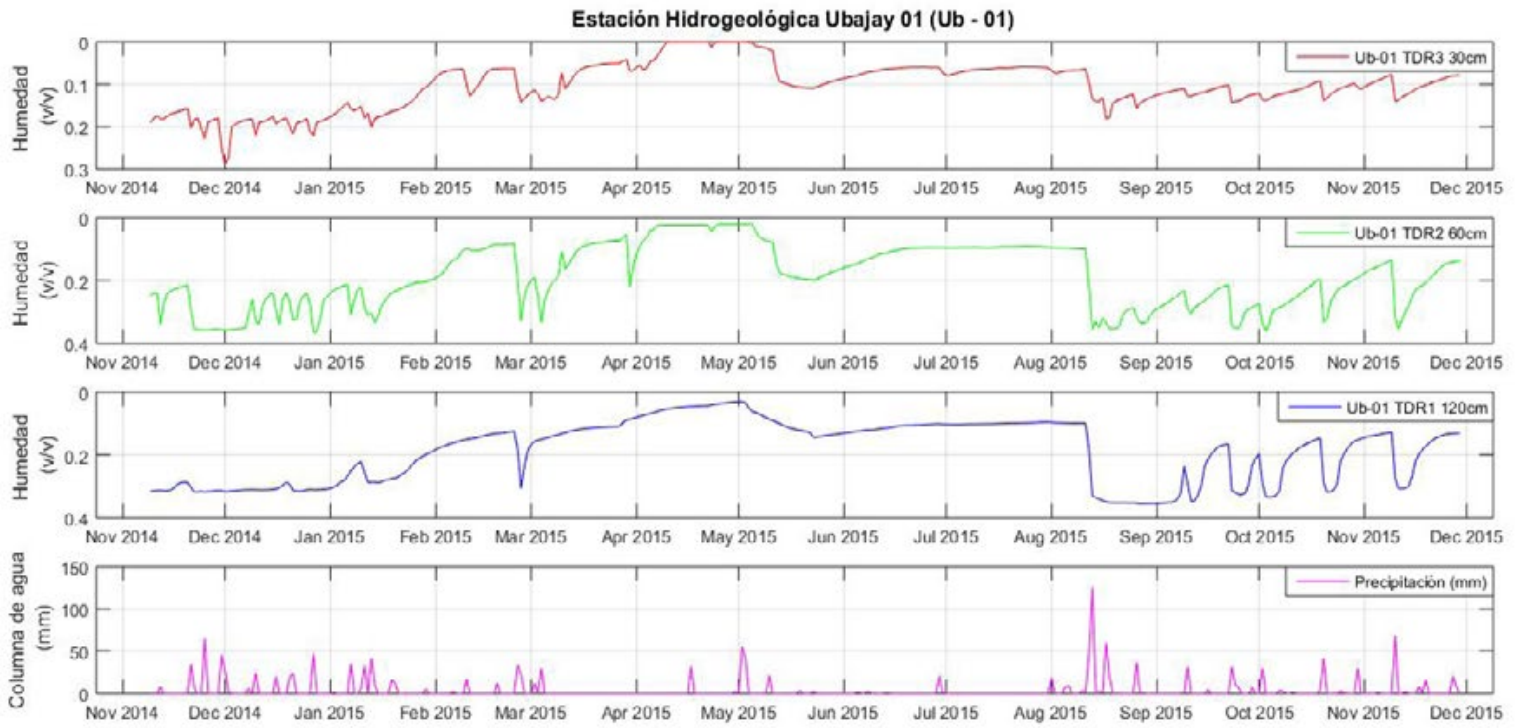

Figura 6. Registro gráfico de humedad de suelo y precipitaciones en la estación Ub-01.

Figure 6. Graphical record of soil moisture and precipitation at the Ub-01 station.
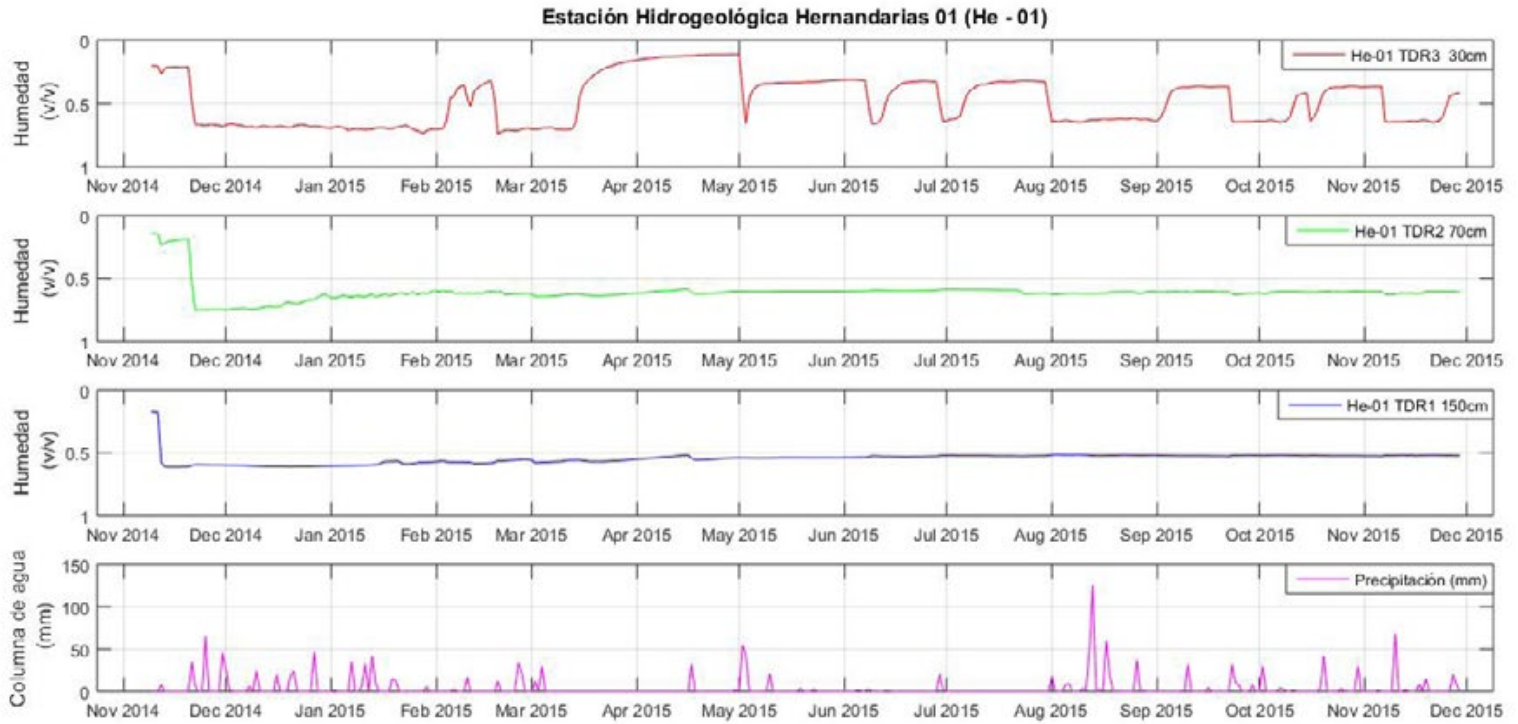

Figura 7. Registro gráfico de humedad de suelo y precipitaciones en la estación He-01.

Figure 7. Graphical record of soil moisture and precipitation at the He-01 station.

La evolución de la $\theta$ cc se presenta en la Tabla 6. Los valores de laboratorio y los valores calculados mediante el método propuesto por Maldonado (2001) son muy similares y validan el empleo de este método cuando no hay medidas directas sobre muestras tomadas en campo. Para el cálculo de los volúmenes de agua transferida como recarga del acuífero, se ha considerado que toda el agua no almacenada en un horizonte se evapotranspira o se transfiere al horizonte inferior, a su vez, se considera que en los horizontes situados bajo el nivel de extracción radicular la evapotranspiración es cero. Además, únicamente el agua que supera el valor de Occ está disponible para ser transferido a horizontes inferiores. De esta forma se contabiliza como recarga neta la porción de las precipitaciones que efectivamente llegan al acuífero libre superior atravesando el suelo.

Los resultados del balance hídrico calculado de esta manera se presentan en la Tabla 7. La primera columna corresponde al excedente de agua que logra pasar cada horizonte de suelo; la columna siguiente contiene 
la relación porcentual del excedente hídrico con respecto a la precipitación total de la serie $(1473,7 \mathrm{~mm})$.

\begin{tabular}{|c|c|c|c|c|}
\hline & $\begin{array}{c}\text { Ub01 } \\
(\text { Lab.) })_{(v / v)}\end{array}$ & $\begin{array}{c}\mathrm{He01} \\
(\text { Lab.) })_{(\mathrm{v} / \mathrm{v})}\end{array}$ & $\begin{array}{l}\text { Ub01 (da- } \\
\text { tos) }{ }_{(\mathrm{v} / \mathrm{v})}\end{array}$ & $\begin{array}{c}\mathrm{He} 01 \text { (da- } \\
\text { tos) } \\
(\mathrm{v} / \mathrm{v})\end{array}$ \\
\hline $\begin{array}{l}\theta_{\text {cc }} \text { hori- } \\
\text { zonte I }\end{array}$ & 0,107 & 0,402 & 0,14 & 0,40 \\
\hline $\begin{array}{l}\theta_{\text {cc }} \text { hori- } \\
\text { zonte II }\end{array}$ & $\mathrm{S} / \mathrm{D}$ & $\mathrm{S} / \mathrm{D}$ & 0,32 & 0,62 \\
\hline $\begin{array}{l}\theta_{\text {cc }} \text { hori- } \\
\text { zonte III }\end{array}$ & $\mathrm{S} / \mathrm{D}$ & $\mathrm{S} / \mathrm{D}$ & 0,31 & 0,60 \\
\hline
\end{tabular}

Tabla 6. Valores de capacidad de campo.

Table 6. Field capacity values.

En la estación Ub-01 el 6,61\% de las precipitaciones logra sobrepasar la zona edáfica $(1,2 \mathrm{~m})$ e incorporarse al agua en tránsito para alcanzar finalmente la zona saturada relativamente somera. Se considera que esta estación es representativa de los suelos desarrollados en la F. Ubajay aflorante en la sección inferior de la cuenca del Arroyo Palmar, una zona fundamentalmente forestal, de escaso uso agrícola y con explotaciones de agua subterránea restringidas al consumo humano y ganadero.

En la estación He-01 la situación es muy diferente, solo el $0,89 \%(13,6 \mathrm{~mm})$ de las precipitaciones sobrepasa la zona edáfica $(1,5 \mathrm{~m})$, además el perfil geológico muestra como la base de la F. Hernandarias está a 26 $\mathrm{m}$ b.b.p. confinando al acuífero Salto Chico, que es el primer nivel saturado, la recarga vertical de este acuí- fero, en la zona de estudio, puede considerarse nula. EI agua debería atravesar 25 metros de niveles arcillosos de muy baja permeabilidad antes de llegar al acuífero.

\begin{tabular}{|l|r|r|}
\hline Intervalo & Excedente $\mathbf{( m m )}$ & Excedente (\%) \\
\hline Ub01 - Horizonte I & 162,98 & 11,06 \\
\hline Ub01 - Horizonte II & 126,96 & 8,61 \\
\hline Ub01 - Horizonte III & 97,38 & 6,61 \\
\hline He01 - Horizonte I & 840,77 & 57,05 \\
\hline He01 - Horizonte II & 154,63 & 10,49 \\
\hline He01 - Horizonte III & 13,16 & 0,89 \\
\hline
\end{tabular}

Tabla 7. Excedente hídrico para cada uno de los horizontes estudiados.

Table 7. Water surplus for each horizon studied.

En las Figuras 8 y 9 se han representado la variación diaria de los excedentes hídricos del suelo para la estación Ubajay y Hernandarias respectivamente. En la estación de Ubajay (Figura 8) se observa una clara estacionalidad y como las lluvias más eficaces, las que producen un excedente medible y recarga, son las que se producen al final del invierno y principio de la primavera, además el flujo de agua parece ser rápido pues existe una cierta correlación en la evolución de la humedad de los tres sensores con un desfase muy corto.

La Figura 9 presenta los datos correspondientes a la estación de Hernandarias. En este caso la estacionalidad solo se observa con claridad en los registradores más profundos mientras que el situado próximo a la

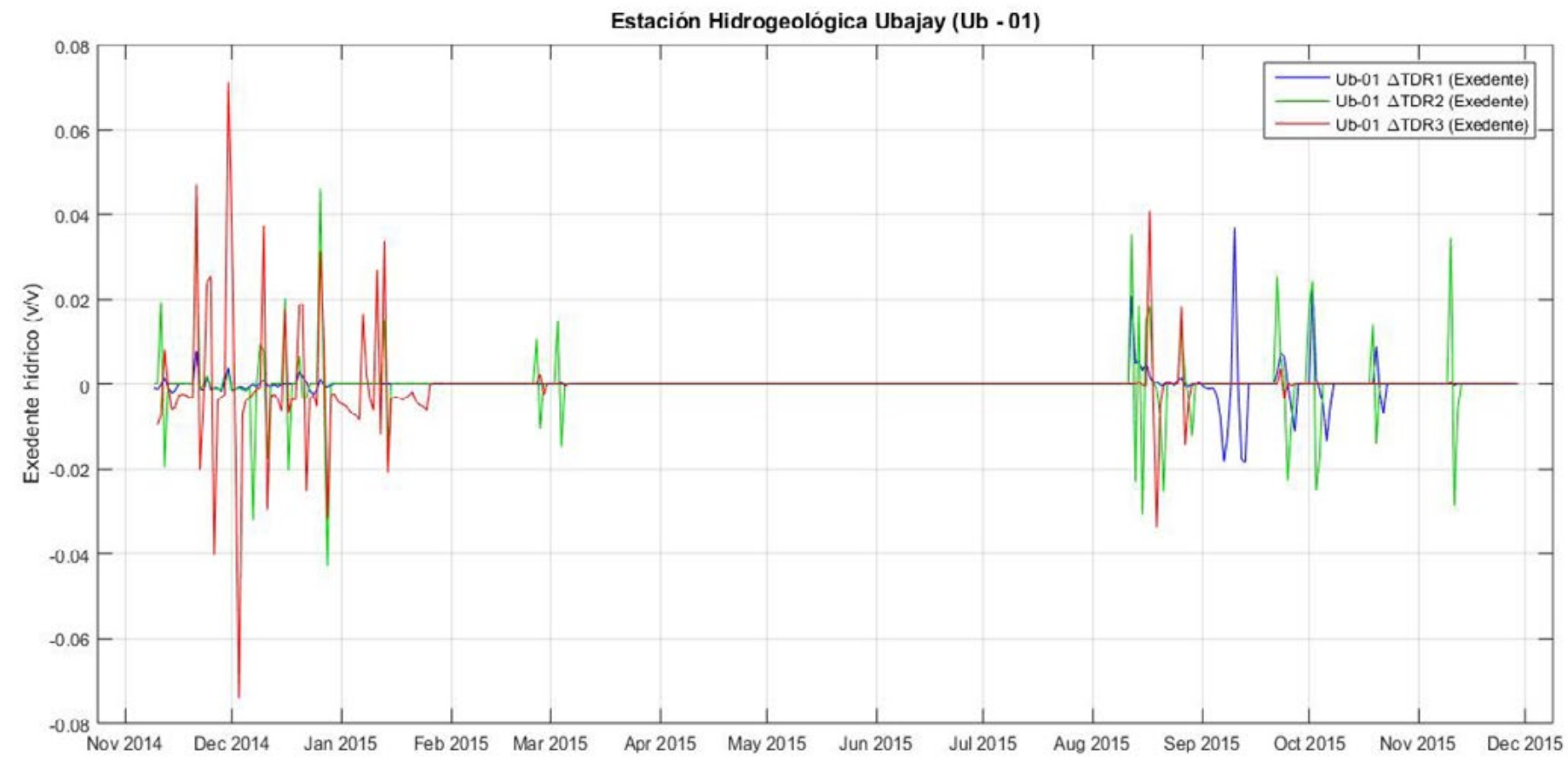

Figura 8. Registro gráfico de excedentes hídricos en la estación Ub-01.

Figure 8. Graphical record of water surplus at the Ub-01 station. 


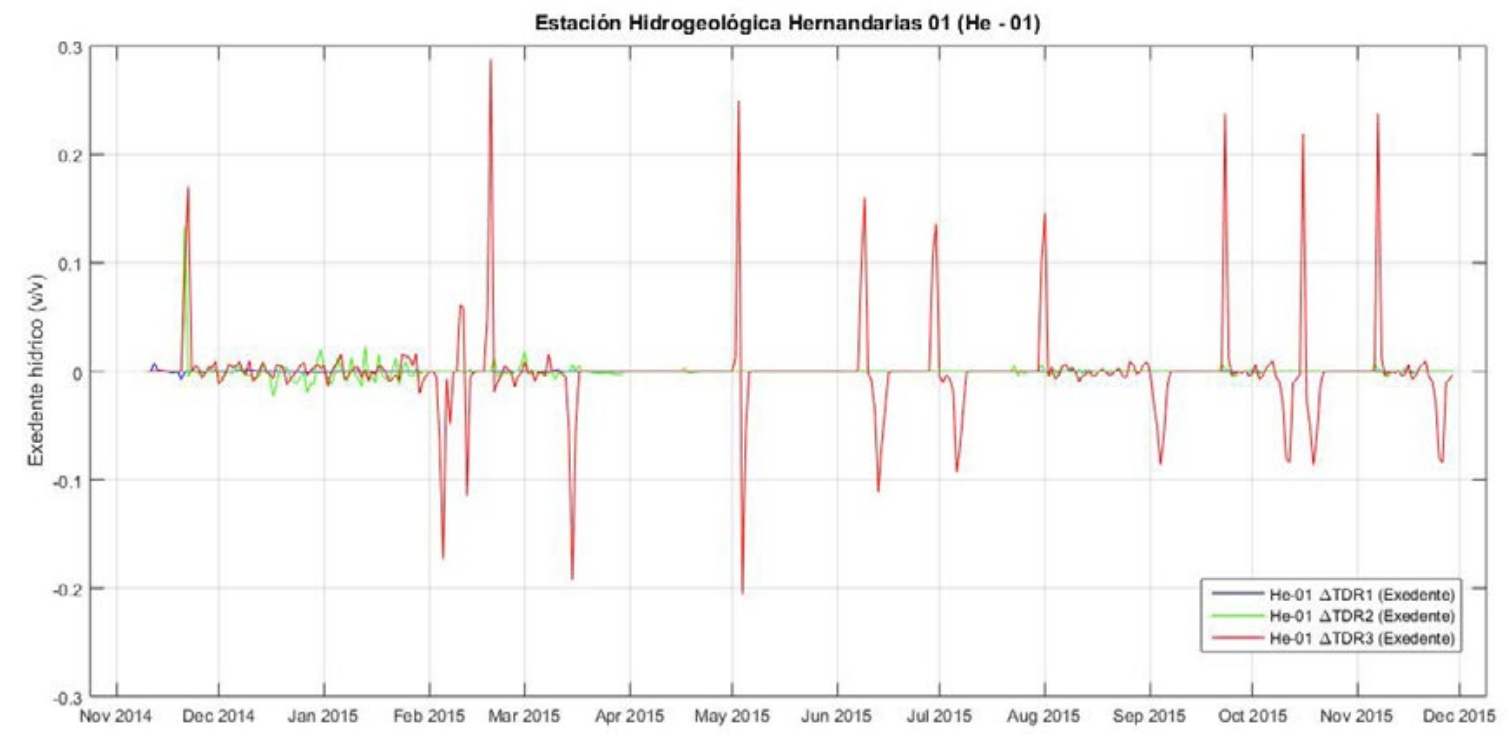

Figura 9. Registro gráfico de excedentes hídricos en la estación He-01. Figure 9. Graphical record of water surplus at the He-01 station.

superficie (TDR3 a 0,3 m) muestra grandes oscilaciones, especialmente significativas durante el periodo seco. Esto se puede interpretar como consecuencia de la existencia de grietas de desecación que permiten el acceso muy rápido del agua al interior del perfil, incluso si la lluvia es de muy escasa entidad. Eso también explica porque muchos de los picos de subida rápida de la humead van seguidos de un descenso igual de rápido, la misma grieta que permite entrar el agua de forma instantánea, si no se cierra lo suficiente, permite la desecación del horizonte también a gran velocidad. El proceso explicado ya fue descrito por Auge y Santi (2002), pero los datos observados indican que su importancia cuantitativa en la recarga del acuífero Salto Chico es muy inferior a lo que proponen.

En la Tabla 8 se presenta la caracterización física de los suelos en estudio (textura, capacidad de campo y punto de marchitez permanente, Ks). El valor de Ks se ha calculado mediante el programa Rosetta (Schaap et al 1998). Como se puede ver, en el conjunto del perfil, las diferencias de conductividad hidráulica previstas por Roseta difieren en aproximadamente un orden de magnitud $\left(0,122 \mathrm{md}^{-1}\right.$ en Ub-01 Horizonte III, frente a $0,01 \mathrm{md}^{-1}$ en He-01 Horizonte II). Esto coincide dentro de los rangos típicos de permeabilidad para este tipo de suelos calculados en Díaz et al (2003).

Por su parte, la aplicación del método de Reynolds y Elrick, (1985), que evalúa la Ks del conjunto de la columna edáfica, ofrece valores de 0,94 $\mathrm{md}^{-1}$ para Ub-01 y $0,0022 \mathrm{md}^{-1}$ para $\mathrm{He}-01$.

Los perfiles de $\theta$ r y $\theta$ s y la conductividad hidráulica saturada (Figura 10) muestran que ambos parámetros son mayores en los primeros $0,3 \mathrm{~m}$ del perfil (horizonte I) cayendo en profundidad casi en un orden de magnitud; esto es esperable pues, además de las diferencias texturales, con la profundidad disminuye la porosidad secundaria y con ello la permeabilidad.

Según algunos autores (Auge y Santi 2002; Santi

\begin{tabular}{|l|r|r|r|r|r|r|r|r|r|}
\hline & \multicolumn{4}{|c|}{ Características sedimento } & \multicolumn{3}{c|}{ Rosetta (Schaap, 1998) } \\
\hline & $\begin{array}{c}\text { Arena } \\
(\%)\end{array}$ & Limo $(\%)$ & $\begin{array}{c}\text { Arcilla } \\
(\%)\end{array}$ & $\begin{array}{c}\delta \\
\mathrm{g} / \mathrm{cm}^{3}\end{array}$ & $\begin{array}{c}\theta \mathrm{s} \\
\mathrm{v} / \mathrm{v}\end{array}$ & $\begin{array}{c}\mathrm{PMP} \\
\mathrm{v} / \mathrm{v}\end{array}$ & $\begin{array}{c}\theta \mathrm{r} \\
\mathrm{v} / \mathrm{v}\end{array}$ & $\begin{array}{c}\theta \text { s } \\
\mathrm{v} / \mathrm{v}\end{array}$ & $\begin{array}{c}\mathrm{Ks} \\
\mathrm{md}^{-1}\end{array}$ \\
\hline Ub-01 Horizonte I & 60,0 & 30 & 10 & 1,41 & 0,14 & 0,04 & 0,020 & 0,38 & 0,723 \\
\hline Ub-01 Horizonte II & 12,00 & 48 & 40 & 1,39 & 0,32 & 0,23 & 0,105 & 0,45 & 0,128 \\
\hline Ub-01 Horizonte III & 52,00 & 27,0 & 21 & 1,44 & 0,31 & 0,09 & 0,043 & 0,40 & 0,122 \\
\hline He-01 Horizonte I & 14,60 & 45,20 & 40 & 1,35 & 0,40 & 0,24 & 0,121 & 0,49 & 0,110 \\
\hline He-01 Horizonte II & 12,50 & 40,00 & 47,5 & 1,31 & 0,62 & 0,28 & 0,112 & 0,57 & 0,010 \\
\hline He-01 Horizonte III & 6,50 & 34,50 & 59 & 1,28 & 0,60 & 0,31 & 0,094 & 0,57 & 0,015 \\
\hline
\end{tabular}

Tabla 8. Textura y características hidráulicas medidas en campo y en laboratorio. (Rosetta, Schaap et al 1998).

Table 8. Texture and hydraulic characteristics measured in the field and in the laboratory. (Rosetta, Schaap et al 1998). 

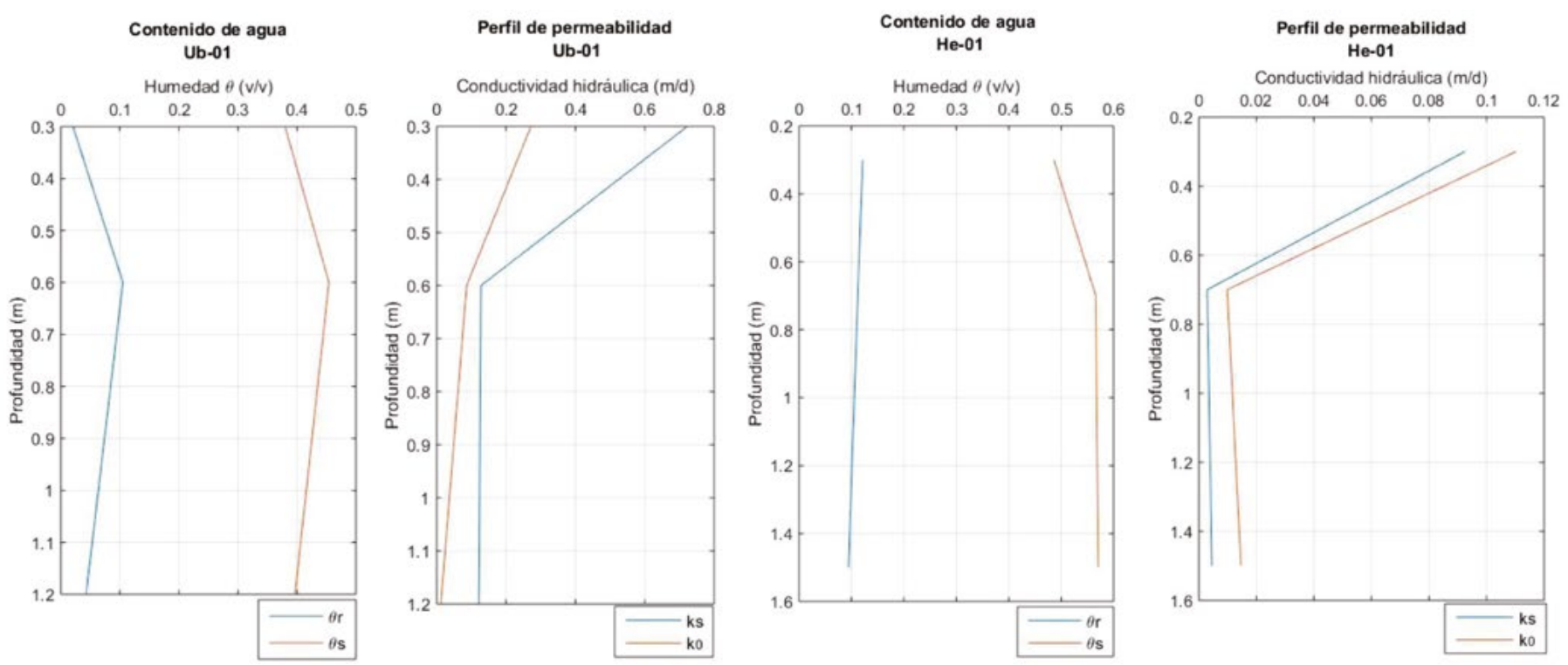

Figura 10. Perfil de saturación de agua y conductividad en Ub-01 y He-01.

Figure 10. Water saturation and conductivity profile in Ub-01 and He-01.

2002) en esta zona la recarga vertical es el principal componente del flujo de entrada de agua al acuífero. Como se deduce de los valores de recarga calculados en este trabajo este dato es discutible pues no es compatible con el exceso de agua en el suelo, que sobre todo en la F. Hernandarias es próximo a cero, incluso tampoco con la poca respuesta de los niveles del acuífero en los periodos de lluvia. Para la estación He-01 el excedente anual del horizonte más profundo (Tabla 7$)$ es de $13.16 \mathrm{~mm}(0.89 \%$ de las precipitaciones). En el caso de la estación Ub-01 el excedente anual del horizonte más profundo (Tabla 7) es de 97,38 $\mathrm{mm}(6,61 \%$ de las precipitaciones). La producción de este excedente se concentra en los meses de invierno y primavera. Calculada la recarga neta (Tabla 9) por el método de Lernner (1990) el valor de la misma resulta aún inferior, de $20,79 \mathrm{~mm}$, es decir el $1,41 \%$ de las precipitaciones para UB-01 y de 0,65 mm, es decir el $0,04 \%$ de las precipitaciones para $\mathrm{He}-01$ (prácticamente nula). Las Figuras 11 y 12 muestran la oscilación de las cargas hidráulicas, o sea diferencia de carga registrada a partir de los cambios de los niveles estáticos.
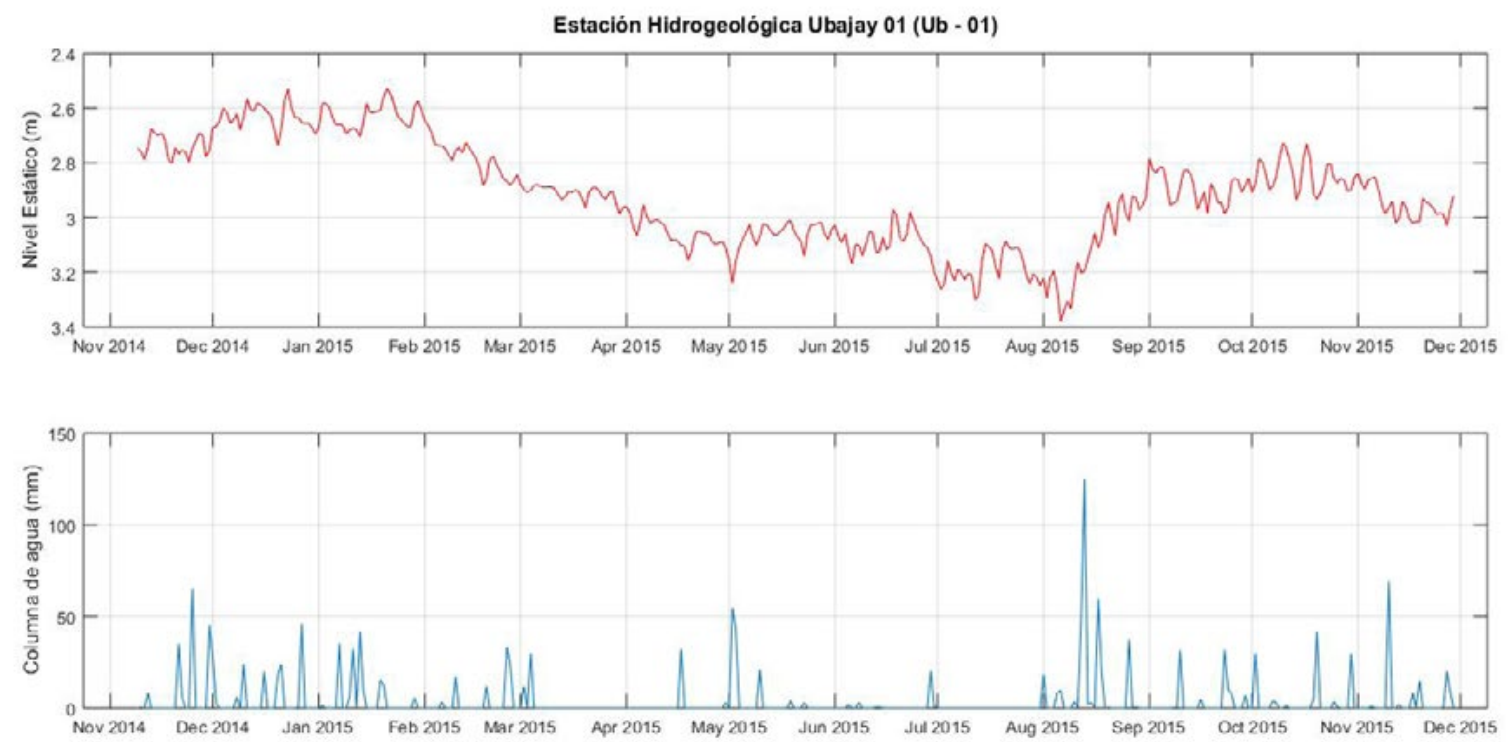

Figura 11. Registro gráfico de nivel estático y precipitaciones en la estación Ub-01.

Figure 11. Graphical record of static level and precipitation at the Ub-01 station. 
Esta oscilación posee mayor amplitud en el piezómetro Ub-01 que en el He-01. Para apreciar mejor las diferencias entre ambas estaciones la Figura 13 muestra comparativamente los registros de cargas hidráulicas donde se observa cuantitativamente la mayor amplitud de oscilación de UB-01 con respecto a He-01. Los resultados obtenidos a partir del cálculo de recarga neta se presentan en la Tabla 9.

\begin{tabular}{|l|l|l|l|l|}
\hline Estación & $\begin{array}{c}\text { Coef. alma- } \\
\text { cenamiento } \\
\text { (adim) }\end{array}$ & $\begin{array}{l}\text { Amplitud } \\
\Delta \mathbf{N e}(\mathbf{m})\end{array}$ & $\mathbf{R n}(\mathbf{m m})$ & $\mathbf{R n}(\%)$ \\
\hline Ub-01 & 0,12 & 0,0328 & 20,79 & 1,41 \\
\hline $\mathrm{He}-01$ & 0,009 & 0,005 & 0,65 & 0,04 \\
\hline
\end{tabular}

Tabla 9. Datos de recarga neta en las estaciones hidrogeológicas. Table 9. Recharge data at the hydrogeological stations.

Una mayor amplitud es una característica relacionada con la posibilidad de recarga vertical sobre todo si se considera las zonas de emplazamiento de la estaciones y en particular la He-01 (alejada de cauces fluviales). Aunque el almacenamiento también tiene relación el trabajo de Masú et.al., (2011) ofrece valores en la cercanía casi inmediata a la UB-01 lo que refuerza la afirmación sobre la amplitud. La F. Hernandarias es un acuitardo (incluso acuícludo) donde no es posible tener valores de almacenamiento por medio de los ensayos hidráulicos. No obstante, los datos de He-01 acusan una menor amplitud de oscilación lo que marca un gran contraste con respecto a la estación Ub-01.

Si se observan los valores de recarga neta (Tabla 9) resultan ser menores que el exceso de agua calcula- do anteriormente. Es posible que este exceso de agua esté incluso sobrevalorado. Una causa probable es que al encontrarse en el límite de cuantificación instrumental haya habido lecturas por exceso.

\section{Conclusiones}

Los estudios disponibles hasta el momento consideraban la infiltración directa del agua de lluvia como la principal fuente de recarga del acuífero Salto Chico situándola entre un máximo del $15 \%$ (Auge y Santi 2002; Auge et al. 2005) y un mínimo del $7 \%$ (Santi 2002; Santi et al. 2009) de las precipitaciones. A lo largo de los años, estos valores se han empleado como referencia para la gestión y planificación de las reservas disponibles del recurso del acuífero entrerriano. Sin embargo, los resultados presentados en este trabajo muestran que en realidad la recarga vertical no es la principal entrada al acuífero y que los valores estimados en anteriores estudios sobreestiman notablemente este componente del balance hídrico. Resulta evidente que deberá estudiarse la participación de otros esquemas conceptuales de recarga que, a manera de hipótesis, pueden involucrar flujos más distales o recargas desde los arroyos interiores que por erosión estén en contacto con las F. Salto Chico permitan la recarga bajo ciertas condiciones de carga hidráulica. Este escenario hace pensar que la recarga vertical no es un proceso dominante ni cuantitativamente importante en la recarga del acuífero Salto Chico. Se trata de un escenario atípico con relación a lo que ocurre en otras zonas de la región pampeana de llanura donde la recarga vertical es el proceso más significativo en la recarga neta de los acuíferos.
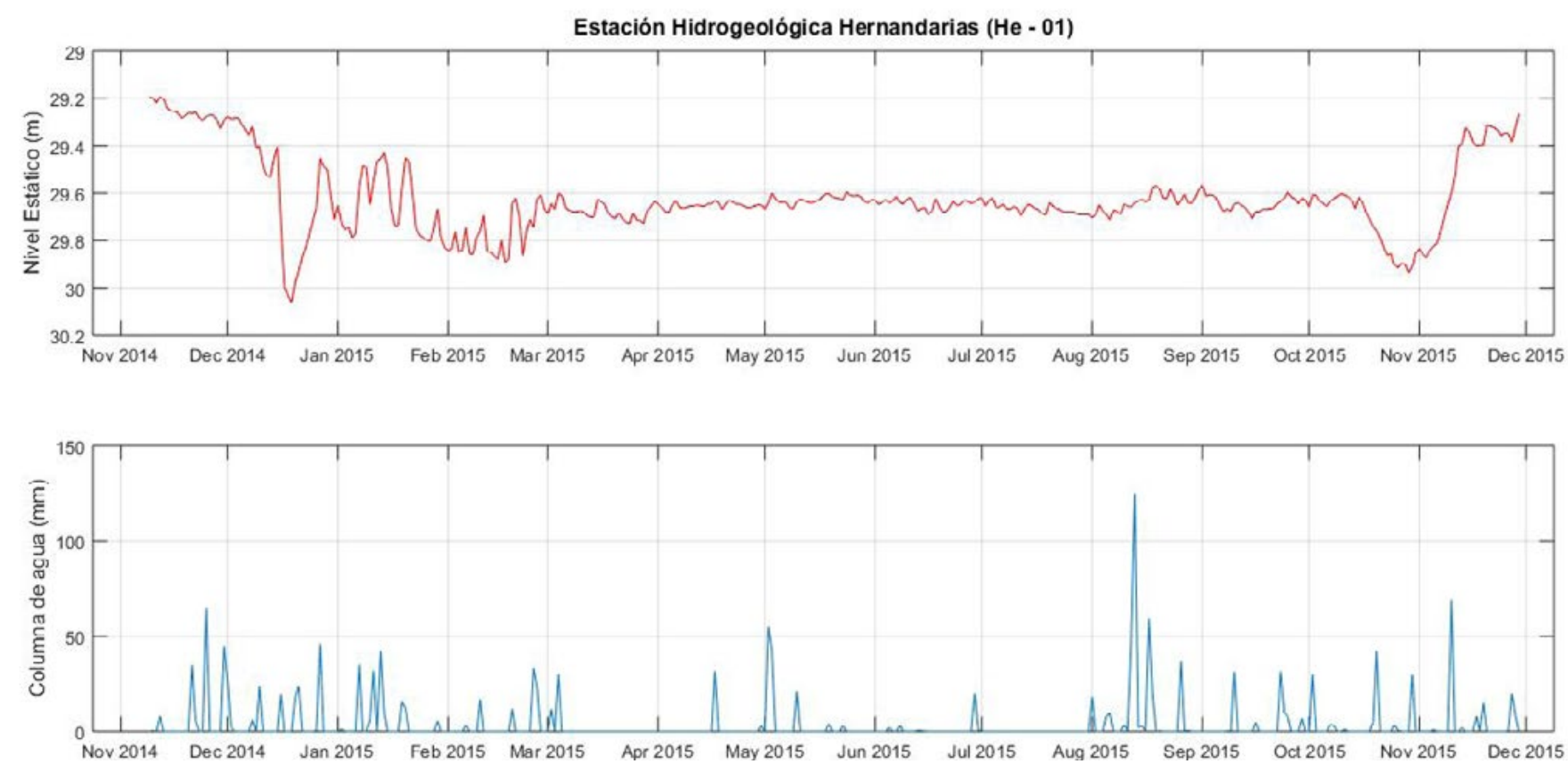

Figura 12. Registro gráfico de nivel estático y precipitaciones en la estación He-01.

Figure 12. Graphical record of static level and precipitation at the He-01 station. 


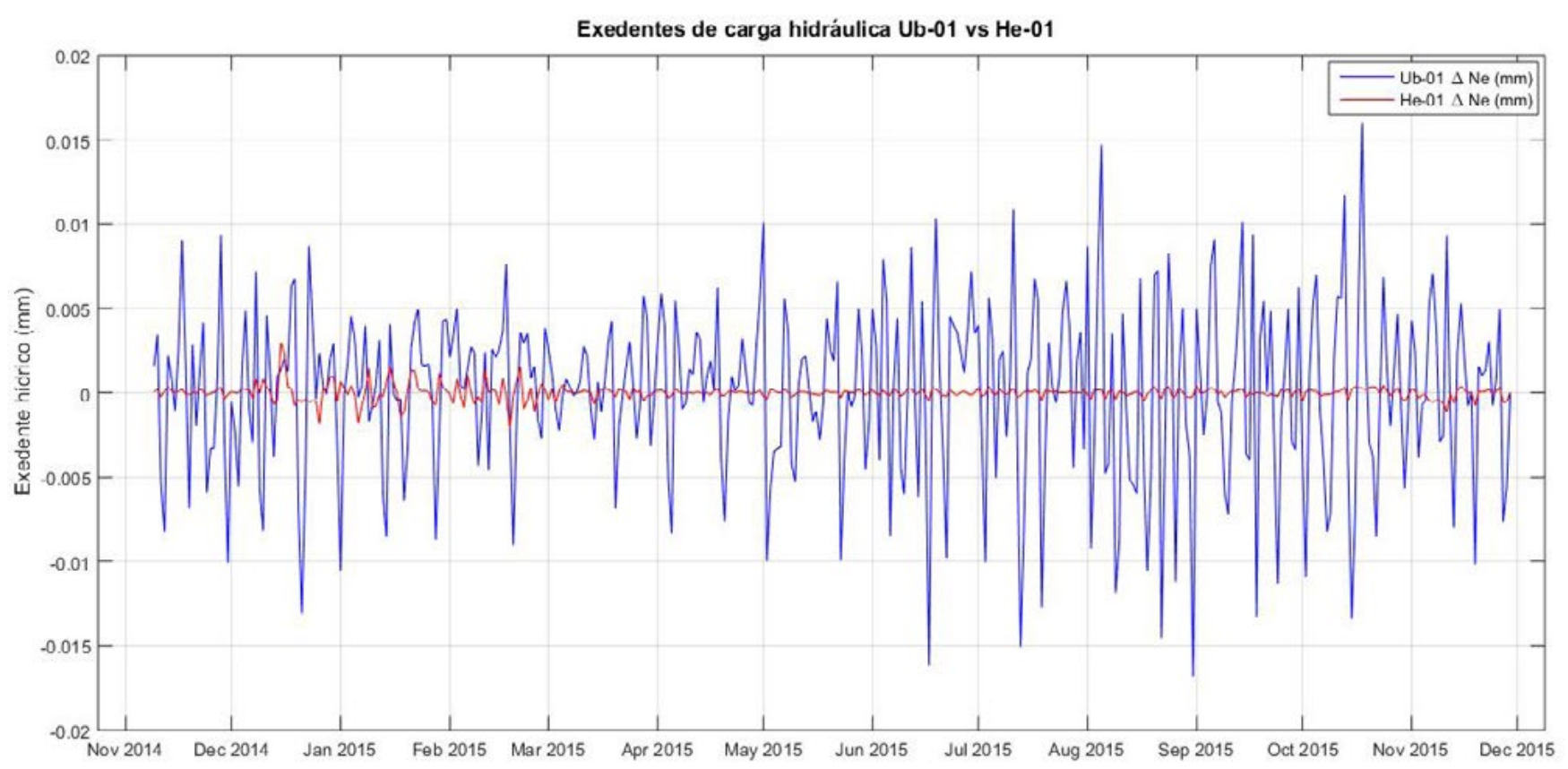

Figura 13. Excedentes de carga hidráulica comparada entre Ub-01 y He-01.

Figure 13. Hydraulic potential surpluses Ub-01vesus He-01.

Para llegar a estas conclusiones se ha tomado la cuenca del Arroyo Palmar como caso estudio detallado. Se han instrumentado dos estaciones de control midiendo parámetros hidrogeológicos (precipitaciones, humedad de suelo y cargas hidráulicas en el acuífero) diferenciando las dos zonas (F. Ubajay y F. Hernandarias) con geología y suelos más representativos en extensión pues entre ambas ocupan más del $95 \%$ de la cuenca, la F. Hernandarias ocupa una extensión aproximadamente tres veces mayor que la F. Ubajay.

En la F. Ubajay se han medido un exceso de agua que llega más allá de la zona radicular que corresponde al $6,61 \%$ de las precipitaciones; en esta formación la recarga neta se ha estimado en el $1,41 \%$ de la precipitación total. En esta zona hay un Parque Nacional donde el uso del suelo (fuera de la reserva) es forestal, y la demanda de agua subterránea es baja porque casi no se practica la agricultura y sobre todo, no se siembra arroz lo que excluye la práctica de riego.

La F. Hernandarias es diferente y sus características no favorecen la percolación de los excedentes hídricos quedando estos retenidos en sus niveles arcillosos sin alcanzar el acuífero Salto Chico. La percolación del agua de lluvia más allá de la zona radicular alcanza el $0,89 \%$ de las precipitaciones y la recarga neta el $0,04 \%$, por lo que es prácticamente nula. Esta zona es más extensa que la anterior, el uso de suelo es agrícola y la demanda de agua subterránea es alta dado que es la zona arrocera donde sí se practica el riego. Los resultados modelizados han tenido un buen ajuste con los datos de campo y laboratorio. Los resultados muestran conductividades hidráulicas (Ks) entre 0,122
- 0,723 m/d para la F. Ubajay y $0,015-0,110 \mathrm{~m} / \mathrm{d}$ para la F. Hernandarias encontrándose dentro de los rangos previstos por Díaz et al., (2003) y siendo concordantes con el resultado del balance de este estudio.

Este cambio en el modelo conceptual de recarga acuífera, que puede ser común a gran parte de la región agrícola productiva de la comarca entrerriana, debe ser tenido en consideración en las evaluaciones de recarga sostenible realizadas sobre la base de la premisa de la recarga vertical (sobre todo usándolas elevadas recargas propuestas del $7-15 \%$ de las lluvias) y que deberán corregirse. Al mismo tiempo la investigación de este estratégico aspecto ambiental de impacto en la productividad agrícola también deberá tomar un curso consecuente con los resultados expuestos y definir un nuevo modelo de recarga acuífera en paridad con los datos medidos y buscando metodologías de evaluación y cuantificación de la recarga diferentes de las empleadas hasta ahora.

\section{Agradecimientos}

Este trabajo se enmarca dentro de los resultados del convenio entre el Instituto Geológico y Minero de España y la UniversidadTecnológica Nacional de Argentina.

\section{Referencias bibliograficas}

Aldonza, J., 2016. Regionalización de lluvias máximas diarias en la Provincia de Entre Ríos, Centro de Estudios y Tecnología del Agua, Facultad de Ciencias 
Exactas, Físicas y Naturales Universidad Nacional de Córdoba. Inédito. Córdoba, Argentina, 104 págs.

Auge, M. Sánchez, C. Santi, M., 2005. Hidrogeología de la región arrocera de Entre Ríos, IV Congreso Hidrogeológico Argentino, Río Cuarto, Córdoba, Argentina. Tomo I, 25-36.

Auge M. y Santi A., 2002. Disponibilidad de Agua Subterránea para Producción Arrocera de la Provincia de Entre Ríos, CFI (Consejo Federal de Inversiones) y Dirección Provincial de Hidráulica de Entre Ríos, Buenos Aires, Argentina, 53pp.

Bertolini, J., 1995. Mapa Geológico de la Provincia de Entre Ríos, República Argentina, (1:500.000). Secretaría de Minería, Dirección Nacional del Servicio Geológico, Buenos Aires, $1 \mathrm{pp}$.

CFI, 1962. Evaluación de los Recursos Naturales de la Argentina. Recursos Hidráulicos Subterráneos. Consejo Federal de Inversiones (CFI), Buenos Aires, Argentina. Tomo V, Vol. I, 463pp.

Cordiní, I., 1949. Contribución al Conocimiento de la Geología Económica de la Prov. de Entre Ríos, Ministerio de Industria y Comercio, Buenos Aires, Argentina. Anales II, MIC 87, 109pp.

D'Orbigny, A., 1842. Voyage dans I'Amerique Meridionale. Pitoislevrautet et cie. Paleontologie Volume 3, Paris, France.

De Alba, E. y Serra N., 1959. Aprovechamiento del Río Uruguay en la zona de Salto Grande, Informe sobre las condiciones y características geológicas. Dirección Nacional de Geología y Minería, Buenos Aires, Argentina. Anales 11(1b), 32pp.

Díaz, E., O. Duarte, J. Cerana y P. Fontanini, 2003. Ajuste metodológico en la medición de la conductividad hidráulica saturada "in situ" en suelos vertisoles y entisoles de la República Argentina mediante el permeámetro de Guelph. In: Álvarez Benedí J. y Marinero P. (ed.) Estudios de la Zona No Saturada del Suelo, España. Vol VI, 153-157.

Filí, M, F., Tujchneider, O., Pérez M. y Paris M., 1993, Investigaciones Geohidrológicas de la Provincia de Entre Ríos, Temas Actuales Sobre Agua Subterráneas, Mar del Plata, Buenos Aires, Argentina. Actas I, 299-315.

Gentile, C, y H, Rimoldi, 1979. Mesopotamia, Academia Nacional de Ciencias, Segundo Simposio Geología Regional Argentina, Córdoba, Argentina, Tomo I, 185-223.

Hausen, J., 1919, Contribución al estudio de la petrografía del Territorio Nacional de Misiones, Dirección General de Minas, Geología e Hidrología, Buenos Aires, Argentina. Boletín $N^{\circ} 21$ Serie B (Geología), $39 p p$

Healy, R. W. and Cook, P. G., 2002. Using groundwater levels to estimate recharge. Hydrogeology Journal. Vol 10, No 1, 91-109.

INTA, 1990. Atlas de suelo de la República Argentina, Instituto Nacional de Tecnología Agropecuaria, Buenos Aires, Argentina. Tomo II, $677 \mathrm{pp}$

INTA, 2002. Carta de Suelos de la República Argentina, Departamento Colón, Provincia de Entre Ríos, Ins- tituto Nacional de Tecnología Agropecuaria (INTA), EEA Paraná, Serie Relevamiento de Recursos Naturales $\mathrm{N}^{\circ} 21,242 \mathrm{pp}$.

INTA, 1999. Carta de Suelos de la República Argentina, Departamento San Salvador, Provincia de Entre Ríos, Instituto Nacional de Tecnología Agropecuaria (INTA), EEA Paraná, Serie Relevamiento de Recursos Naturales $N^{\circ} 18,166$ pp.

Iriondo, M. H. 1996, Estratigrafía del Cuaternario de la Cuenca del Río Uruguay, XIII Congreso Geológico Argentino y III Congreso de Exploración de Hidrocarburos, Buenos Aires, Argentina, Tomo IV, 15-25.

Iriondo, M. H. 1980. El Cuaternario de Entre Ríos, Revista Asociación Ciencias Naturales del Litoral, Corrientes, Argentina. Vol, 11, 125 - 141.

Lerner, D., N., 1990. Techniques. In: van Acken R. (ed.). Groundwater recharge. A guide to understanding the natural recharge, Hannover, Germany, 99-229.

Machado, P. Silva Busso, A., Chury, M., Salarí, A., Ojeda, C., 2017. Determinación de Parámetros Hidrogeomorfológicos de la Cuenca del Arroyo Palmar Aplicando Técnicas de Teledetección, VI Congreso Bianual PROIMCA y IV Congreso Bianual PRODE$C A$, Bahía Blanca, Argentina, Actas en $C D, N^{\circ} 23$, 1-12.

Maldonado, T., 2001. Manual de riego parcelario. Oficina regional de la FAO para América Latina y el Caribe, Santiago, Chile, 130pp.

Masú, J., Silva Busso, A. y Amato, S., 2011. Aspectos Geológicos del Acuífero Salto Chico en la Cuenca del Arroyo El Palmar, Provincia de Entre Ríos, República Argentina, VII Congreso Argentino de Hidrogeología y V Seminario Hispano Latinoamericano Sobre Temas Actuales de la Hidrología Subterránea, Salta, Argentina, Actas en CD, 56 - 60

Mualem, Y., 1976. A new model predicting the hydraulic conductivity of unsaturated porous media. Water Resourse. Res. (12), 513-522.

Micou, M. P., 2003, Riesgo ambiental por invasiones biológicas en una zona con alto valor de conservación. Departamento de Geografía, Facultad de Filosofía y Letras, Universidad Buenos Aires, Inédito. Buenos Aires, Argentina, 157 págs.

Reig, O., 1956, Sobre la posición sistemática de "Zygolestes paranensis" Amegh, y de "Zygolestes entrerrianus" Amegh, Revista Holmbergia, N ${ }^{\circ} 5$ (1213), 209-226.

Reynolds, W., Elrick, D., 1985. In situ measurement of field-saturated hydraulic conductivity, sorptivity, and the $\alpha$-parameter using the Guelph permeameter. Soil Science Journal. Volume 140 (4), 292-302.

Richards, L. and Weaver, L., 1944. Moisture retention by some irrigated soils as related to soil moisture tension. Journal of Agricultural Research $N^{\circ} 69$, 215-235.

Rojas, A. y Saluso, J., 1987. Informe climático de la provincia de Entre Ríos, Instituto Nacional de Tecnología Agropecuaria EEA-Paraná, Publicación Técnica $\mathrm{N}^{\circ} 14,20 \mathrm{pp}$. 
Santi, M., 2002. Estudio de Aguas Subterráneas en el Sudoeste de la Provincia de Entre Ríos. Consejo Federal de Inversiones (CFI), Buenos Aires, Argentina, $76 \mathrm{pp}$.

Santi, M., Bianchi G., y Rezzónico, G., 2009. Agua subterránea en el noreste de Entre Ríos, VI Congreso Argentino de Hidrogeología, Planificación y gestión de aguas subterráneas Santa Rosa, La Pampa, Argentina, Acta I, 63-73.

Schaap, M., Leij, F. and van Genuchten, M., 1998. Neural network analysis for hierarchical prediction of soil water retention and saturated hydraulic conductivity. Soil Science. Society. American. Journal. $N^{\circ} 62,847-855$.

Silva Busso, A. y Amato, S., 2013. Posibilidades de Explotación y Demanda Hídrica sobre el Acuífero Ubajay en el Sector Oriental de la Provincia de Entre Ríos, $24^{\circ}$ Congreso Nacional del Agua, San Juan, Argentina, Actas: Tomo 2, 56-66.

Silva Busso, A. y Amato, S. 2017. Depósitos fluviales del Plio-pleistoceno-holoceno de la Provincia de Entre Ríos y sus Implicancias Hidrogeológicas, Revista de la Asociación Geológica Argentina, Vol 74 (3), $338-356$.

Silva Busso, A., y Machado, P., 2019. Aspectos Geológicos e Hidrogeológicos del Parque Nacional EI Palmar, Entre Ríos, Argentina, Boletín Geológico Minero de España. Vol 130 (4), 773-788.

Silva Busso, A., 1999. Contribución al Conocimiento
Geológico e Hidrogeológico del Sistema Acuífero Termal de la Cuenca Chacoparanense Oriental Argentina, Departamento de Geología, Facultad de Cs, Exactas y Naturales, Universidad Buenos Aires. Inédito. Buenos Aires, Argentina, 504 págs.

Soil Survey Staff, 1975. Soil Taxonomy. Agriculture Handbook N $N^{\circ} 436$, U. S. Government Printing Office, Washington D. C, USA, 754 pp.

Thornthwaite, C. and Mather, J., 1957. Instructions and tables for computing the potential evapotranspiration and the water balance, Climate Drexel Institute of Technology. $\mathrm{N}^{\circ} 10,185-311$.

Tófalo, O., 1986. Caracterización sedimentológica y estratigrafía de las Formaciones Puerto Yeruá y Arroyo Avalos, provincias de Entre Ríos y Corrientes, Departamento de Geología, Facultad de Cs, Exactas y Naturales, Universidad Buenos Aires.Inédito. Buenos Aires, Argentina, 221 págs.

van Genuchten, M., 1980. A closed-form equation for predicting the hydraulic conductivity of unsaturated soils. Soil Science Society American Journal $N^{\circ} 44,892-898$.

Vitulich, E. B. 1982, Descripción geológica de los Descripción geológica de los depósitos yesíferos de la Provincia depósitos yesíferos de la Provincia de Entre Ríos, región Paraná - La Paz. Departamento de Geología, Facultad de Cs, Exactas y Naturales, Universidad Buenos Aires. Inédito. Buenos Aires, Argentina, 87 págs.

Recibido: julio 2019

Revisado: noviembre 2019

Aceptado: enero 2020

Publicado: diciembre 2021 
